\section{Pacific Northwest}

National Laboratory

Operated by Battelle for the

U.S. Department of Energy

\title{
GridWise Standards Mapping Overview
}

\author{
M. L. Bosquet
}

March 2004

Prepared for the U.S. Department of Energy under Contract DE-AC06-76RL01830

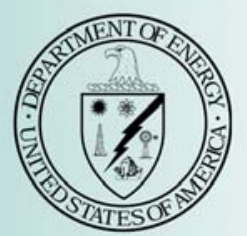




\title{
DISCLAIMER
}

This report was prepared as an account of work sponsored by an agency of the United States Government. Neither the United States Government nor any agency thereof, nor Battelle Memorial Institute, nor any of their employees, makes any warranty, express or implied, or assumes any legal liability or responsibility for the accuracy, completeness, or usefulness of any information, apparatus, product, or process disclosed, or represents that its use would not infringe privately owned rights. Reference herein to any specific commercial product, process, or service by trade name, trademark, manufacturer, or otherwise does not necessarily constitute or imply its endorsement, recommendation, or favoring by the United States Government or any agency thereof, or Battelle Memorial Institute. The views and opinions of authors expressed herein do not necessarily state or reflect those of the United States Government or any agency thereof.

\author{
PACIFIC NORTHWEST NATIONAL LABORATORY \\ operated by \\ BATTELLE \\ for the \\ UNITED STATES DEPARTMENT OF ENERGY \\ under Contract DE-AC06-76RL01830
}

This document was printed on recycled paper. 


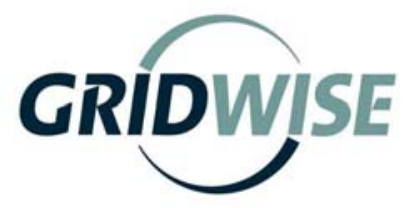

\section{GridWise Standards Mapping Overview}

M. L. Bosquet

March 2004

\author{
Prepared for the \\ U.S. Department of Energy \\ under Contract DE-AC06-76RL01830
}




\section{Summary}

"GridWise $\mathrm{TM}^{\mathrm{TM}}$ is a concept of how advanced communications, information and controls technology can transform the nation's energy system - across the spectrum of large scale, central generation to common consumer appliances and equipment - into a collaborative network, rich in the exchange of decision making information and an abundance of market-based opportunities" (Widergren and Bosquet 2003) - accompanying the electric transmission and distribution system fully into the information and telecommunication age. This report summarizes a broad review of standards efforts are related to GridWise - those that could ultimately contribute significantly to advancements toward the GridWise vision, or those which represent today's current technological basis upon which this vision must build.

Along with an extensive listing of specific standards efforts, this report compiles and synthesizes this information to help readers conceptualize the relative positioning of different standards, or standards-related efforts. ${ }^{1}$ A clearer picture of the GridWise standards 'landscape' begins to emerge as we consider these numerous efforts from various different stakeholder viewpoints. Figure S-1 illustrates a general categorization of standards efforts related to GridWise. ${ }^{2}$

This report does not provide an exhaustive view of standards surrounding GridWisethose standards efforts are constantly evolving, and there is no simple, accepted view as to what represents a standard or standards development and what does not. Yet, the identification of such an extensive breadth of standards efforts reemphasizes the importance

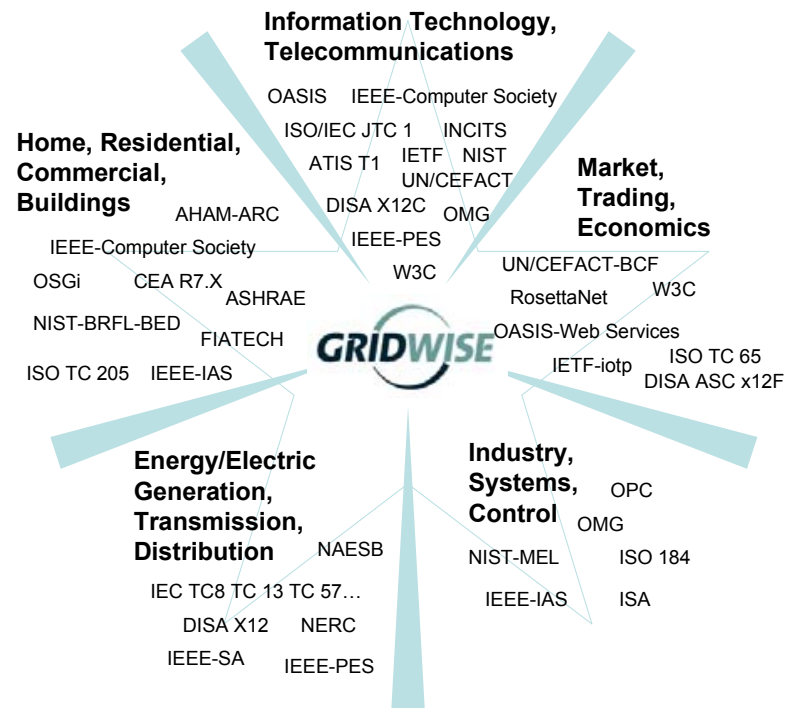

Figure S-1 General categorization of standards efforts related to GridWise of stakeholder involvement and buy-in for progress toward the GridWise vision. A broad, evolving framework, fully spanning and leveraging stakeholders from each of the viewpoints suggested in this report will be needed for effective progress toward realizing GridWise envisaged transformation, which will require mutual acknowledgement between technological fields for development, compatibility, integration, interoperation, convergence, adoption and beyond.

\footnotetext{
${ }^{1}$ In addition to this overview report, separate background draft reports have been compiled providing further, more detailed information on each of the standards developing organizations presented in this overview to serve as condensed introductions for those working on GridWise architecture or related areas.

${ }^{2}$ Section 6.1 lists basic acronyms for these standards organizations and Appendix A provides details on the various efforts and sub-groups. A detailed glossary of acronyms is not yet available.
} 


\section{Acknowledgements}

The author would like to thank Steve Widergren, Landis Kannberg and Rob Pratt of Pacific

Northwest National Laboratory (PNNL) who provided invaluable support and advice throughout all stages of this report and Krishnan Gowri (PNNL) for his contributions to this work. Thanks are also extended to Sue Arey, Joe Roop, David Winiarski and John DeSteese (PNNL) for their editorial assistance and reviewing.

The author would also like to thank the Integrated Energy and Communications Systems Architecture (IECSA) project - a project within the Consortium for Electric Infrastructure to Support a Digital Society (CEIDS) program of the Electricity Innovation Institute (E2I) - whose Task 2 deliverable "A Guide to Existing Communications, Information, and Systems Architecture Technologies" (Sanza and Adamiak, 2002) provided an extensive background compilation for this work. 


\section{Contents}

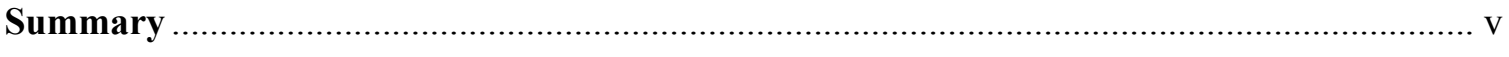

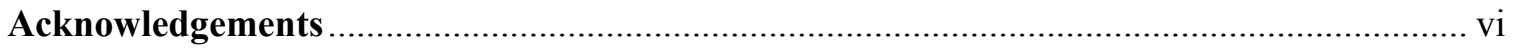

1 Context: Initial Standards Organization Mapping ….......................................... 1

2 Context-Specific Views of GridWise-Related Standards .............................................. 3

2.1 How Do We Define a GridWise-Related Standards Effort? ........................................ 3

2.2 Different Points of View from Different Sectors .................................................. 3

2.3 Results from Views of GridWise-Related Standards ............................................. 11

3 Multi-Dimensional Conceptualizations of this Standards Environment ...................... 13

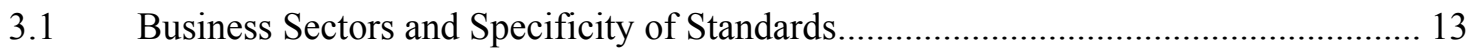

3.2 Mapping Standards Groups vs. Electric Sector Position.............................................. 14

3.3 Functional Levels of Communication and Control Technologies ............................ 16

3.4 Using Functional Levels to Understand and Compare Standards............................ 18

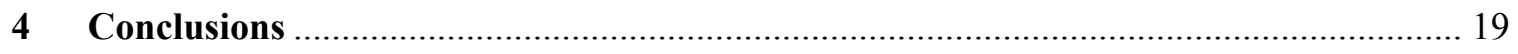

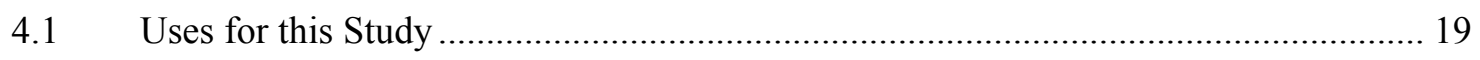

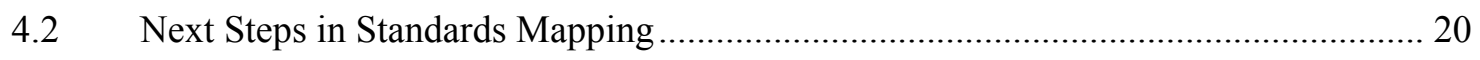

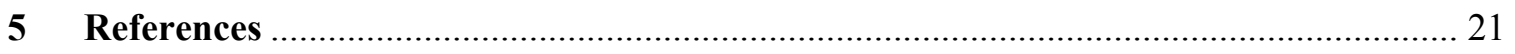

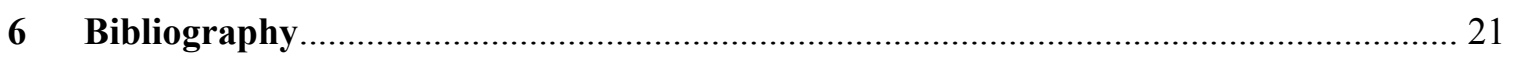

6.1 Main Websites of Standards Developing Organizations Studied ............................... 23

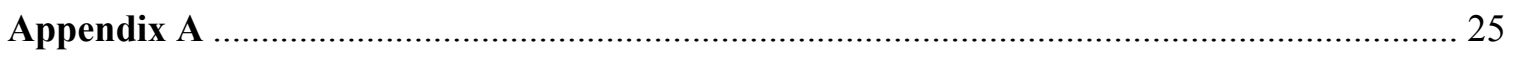

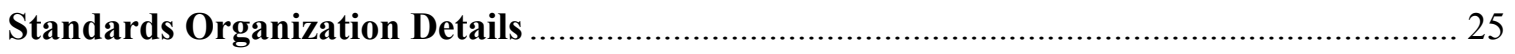

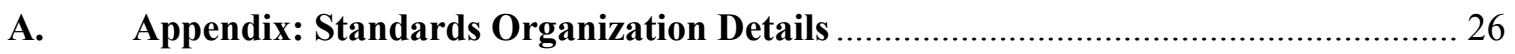

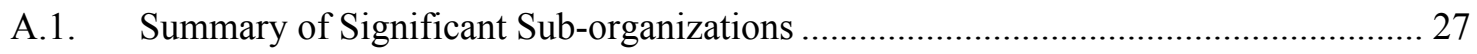

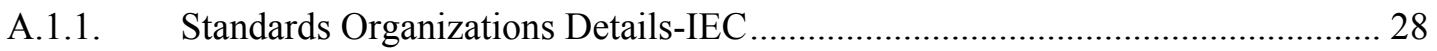

A.1.2. Standards Organizations Details-IEEE............................................................ 30

A.1.3. Standards Organizations Details-NAESB \& NERC …..................................... 31

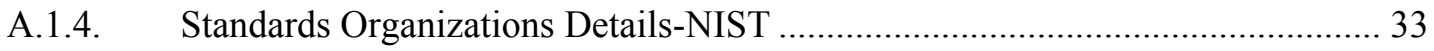


A.1.5. Standards Organizations Details-UN/CEFACT, OASIS \& DISA ....................... 34

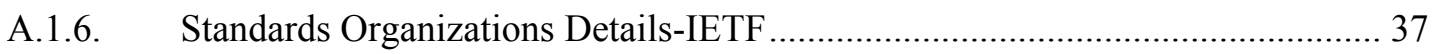

A.1.7. Standards Organizations Details-OMG \& W3C ….............................................. 39

A.1.8. Standards Organizations Details-RosettaNet ...................................................... 41

A.1.9. Standards Organizations Details_-EIA \& CEA \& TIA …...................................... 42

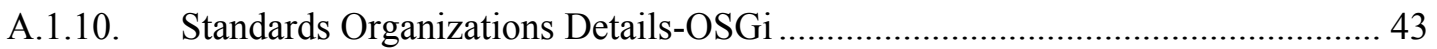

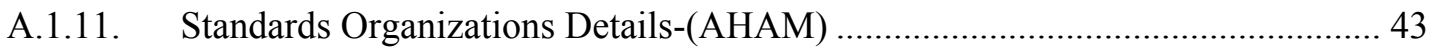

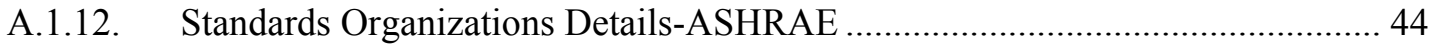

A.1.13. Standards Organizations Details-FIATECH …................................................. 45

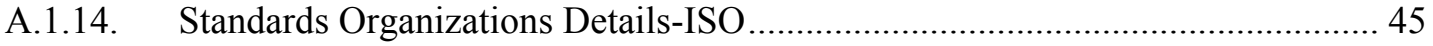

A.1.15. Standards Organizations Details-OPC \& ISA …................................................. 47

A.1.16. Standards Organizations Details-ITI-INCITS .................................................. 48

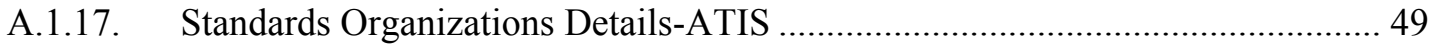

A.1.18. Standards Organizations Details-ANSI ....................................................... 50

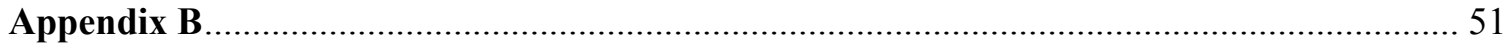

General Background on Standards-Related Organizations ................................................ 51

B. General Background on Standards-Related Organizations ................................. 52

B.1. General Categories of Standards Organizations …................................................... 52

B.2. General Characteristics of Standards-Related Organizations ................................... 53

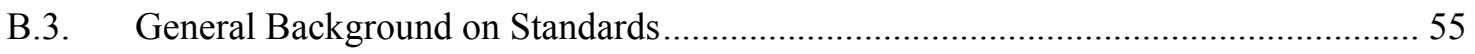

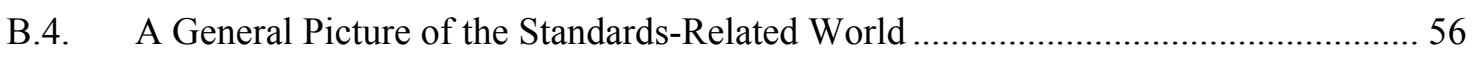

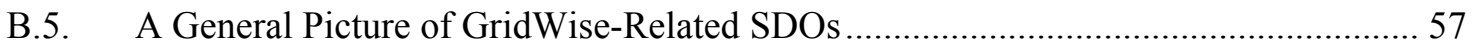




\section{Figures}

Figure S-1 General Categorization of Standards Efforts Related to GridWise ............................. iii

Figure 1-1 General Schema of Standards-Related Organizations ........................................... 1

Figure 1-2 Dividing GridWise stakeholders into Five General Groups ..................................... 5

Figure 3-1 Mapping Groups vs. Business Sectors and Specificity ......................................... 14

Figure 3-2 Mapping Groups vs. Position along Electric Sector Path ....................................... 15

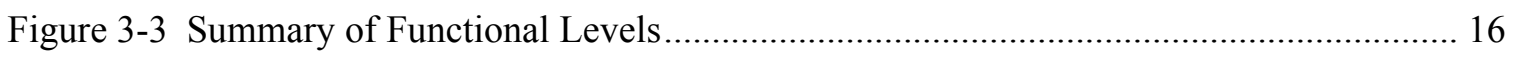

\section{Tables}

Table 2-1 Residential/Commercial/Buildings-Centered View of GridWise-Related Standards

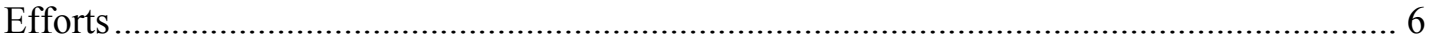

Table 2-2 IT \& Telecommunications-Centered View of GridWise-Related Standards Efforts..... 7

Table 2-3 Market/Trading/Economic-Centered View of GridWise-Related Standards Efforts..... 8

Table 2-4 Industry/Systems/Contro1/Manufacturing-Centered View of GridWise-Related

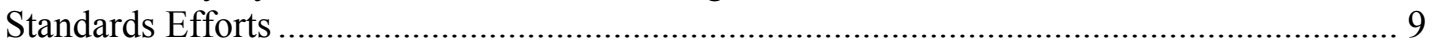

Table 2-5 Energy-Centered View of GridWise-Related Standards Efforts................................ 10

Table 3-1 Functional Levels Surrounding Communication and Control Technologies ................ 17 


\section{Context: Initial Standards Organization Mapping}

This study starts from the basis set forth in the "Initial Standards Organizations Mapping for GridWise Architecting Needs', which provided background information concerning the nature of standards-related organizations, and an initial picture of standards developing organizations (SDOs), which are relevant to the GridWise architecture. These organizations are presented in Figure 1-1.

To improve upon and extend the initial standards mapping, this document synthesizes the subcommittees, design centers, and relevant specific standards of the more significant SDOs and provides mechanisms to better comprehend this complex picture

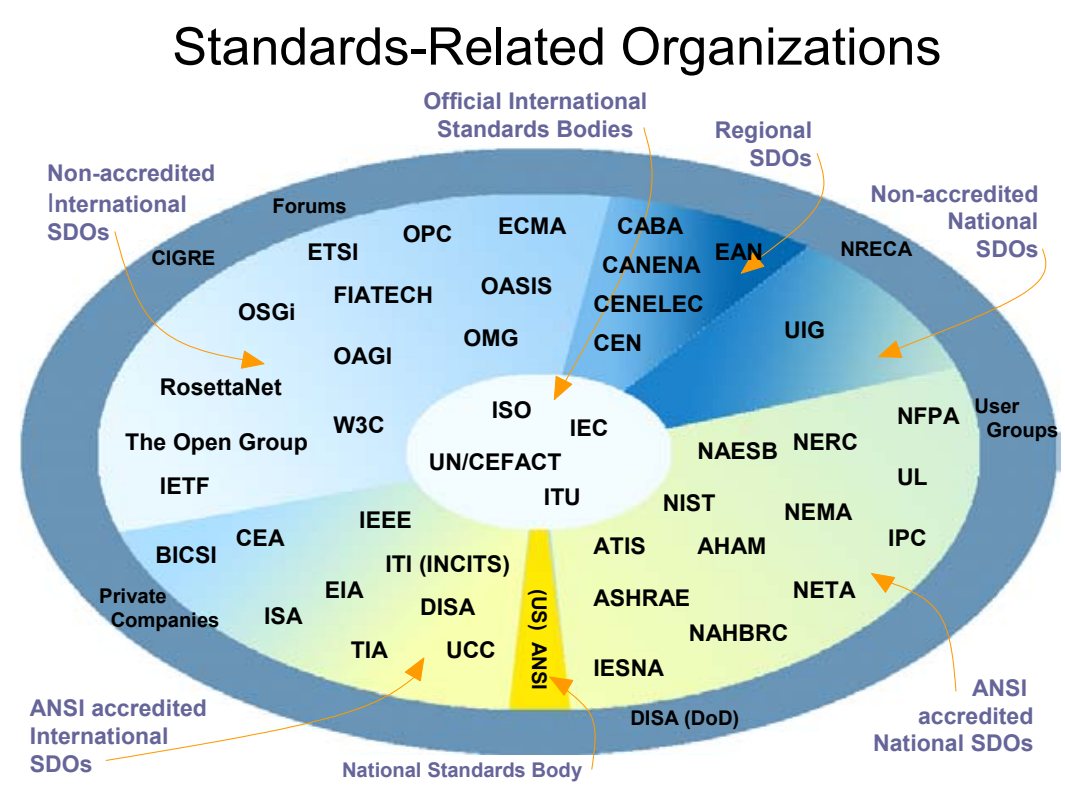

Figure 1-1 General Schema of Standards-Related Organizations as follows:

- Context specific views summarizing GridWise-related standards efforts

- Presentation of multi-dimensional conceptualizations of this standards environment

- Description of functional levels surrounding communication and control technologies and standards

- Appendix A: List of separate working documents compiling details on each SDO; and summaries of the most relevant subcommittees or projects in each key standards organization.

- Appendix B: General background on standards-related organizations, helping clarify what is a standard, what is a standards developing organization, what does it mean to be 'accredited', what characterizes international standards organizations, and how can we generally visualize the standards-related world.

The purpose of this document and the accompanying studies of each significant SDO is two-fold. First, the conceptual views and the wealth of details are intended to provide a solid reference for

\footnotetext{
${ }^{3}$ Appendix B in this report presents a general background discussion on standards organizations extracted from Bosquet, ML. 2003. Initial Standards Organizations Mapping for GridWise Architecting Needs. Draft report, Pacific Northwest National Laboratory, Richland, Washington.

${ }^{4}$ Section 6.1 lists basic acronyms for these standards organizations and Appendix A provides details on the various efforts and sub-groups. A detailed glossary of acronyms is not yet available.
} 
GridWise 'architects' and other technical experts among GridWise stakeholders. Second, this compilation represents an important basis for GridWise standards mapping, creating a more thorough and clear picture of the entire field standards efforts that may be relevant to GridWise and the organizations involved in moving those efforts forward. This should assist the GridWise Architecture Board in understanding where efforts are needed to encourage standards convergence, interoperability, and pivotal progression of new information, communication, and transaction technologies in line with the GridWise vision.

The scope of this study has been limited primarily to standards development organizations that have been identified as being above average in significance to GridWise. Some less significant organizations have also been studied and included where they have subgroups or address specific projects that help provide a more complete picture of GridWise-related standards efforts.

The breadth of this study should be viewed as a starting point - it is in no way intended to be exclusive, limiting or exhaustive. Effective technological advances and standards development efforts are moving targets, so some highly significant efforts related to GridWise may be inadvertently overlooked. Also, trade groups and private organizations were generally not within the scope of this study, although they, too, may host efforts of significance for GridWise technical direction. ${ }^{5}$ Admittedly, there is not always a clear demarcation between standards developing organizations and marketing-oriented trade groups.

\footnotetext{
${ }^{5}$ For example, CABA, the Continental Automated Buildings Association, is an industry association (trade group) that may rival some SDOs in its significance to GridWise architecture efforts, for the stakeholder outreach opportunities it enables, and for its participation in some key standards development efforts.
} 


\section{Context-Specific Views of GridWise-Related Standards}

Establishing a map of GridWise-related standards and standards organizations poses some fundamental problems: What should this map look like? How do we effectively map a 'world of standards' involving multiple dimensions? How do we define GridWise-related? The concept of GridWise is quite new, so the terminology, boundaries, and understanding of the GridWise vision remain somewhat fuzzy.

Because this standards-mapping effort is starting from such a blank slate, we must define the boundaries chosen to define a GridWise-related standards effort, and the methods of mapping that have been employed in the following analyses.

\subsection{How Do We Define a GridWise-Related Standards Effort?}

"GridWise ${ }^{\mathrm{TM}}$ is a concept of how advanced communications, information and controls technology can transform the nation's energy system - across the spectrum of large scale, central generation to common consumer appliances and equipment - into a collaborative network, rich in the exchange of decision making information and an abundance of market-based opportunities" (Widergren and Bosquet 2004). So 'GridWise-related' will include elements that touch on energy systems, including generation, transmission, distribution, and end-use (from residential loads to industry). Technologically, GridWise covers a broad range of communications, information and control technology, with added emphasis on transactions (market exchanges, independent agents, intelligent, distributed decision-making...). GridWise spans several business sectors including: power, information technology, telecommunications, finance and markets, building, and manufacturing.

One basic premise is that 'GridWise-related' standards efforts all pertain directly or indirectly to energy systems, and involve mechanisms to come to agreement on aspects regarding communication, information and controls within and between these extensive systems. The GridWise vision aims to weave together traditional elements of supply and demand, transmission and distribution with new distributed "plug-and-play" technologies using advanced telecommunications, information and control approaches to create a "society" of devices that functions as an integrated, collaborative (transactive) system. Standards efforts identified in this survey are those that could be judged to contribute to this GridWise vision or that present today's current, existing technological basis upon which this vision must build.

\subsection{Different Points of View from Different Sectors}

In cartography, a common two-dimensional Mercator projection of the world can greatly distort the relative surface of areas like Greenland and can provide quite different images of the world depending on whether the projection is centered on North America or on India. Similarly, applying different viewpoints and different mapping projections to GridWise-related standards efforts will effect how this information is interpreted by different readers.

In the field of electronic data interchange (EDI) as in more recent research on the semantic web, experts have been confronted with the difficulties stemming from different viewpoints. A given word like 'price', or a given XML tag like '<price>' may be understood quite differently by different actors who must share the information - with disputes between 'price' and 'cost', 'with tax' and 'after tax', 'local currency' or not, etc. There is no 'right' or 'best' definition, and different actors will continue seeing things in different ways: '...multiple different legitimate and often essential views exist.' (Madnick 2001). 
Research on the evolution of semantics, on the semantic web, and on metadata all underline the importance of context in interpreting information. In this light and given the breadth of contexts with some relevance to GridWise, several different viewpoints are used to present GridWiserelated standards efforts. Each of the following tables presents GridWise-related standards from a different viewpoint, imagining which standards efforts, important to actors in a given sector, would appear to be relevant to GridWise from their own vantage point: ${ }^{6}$

- Home/Residential/Commercial/Buildings

- IT/Telecommunications

- Market/Trading/Economics

- Industry/Systems/Control

- Energy/Electric Generation, Transmission \& Distribution

Each table presents GridWise-related standards efforts which actors in those fields would be likely to be aware of and use. Figure 2-1 presents a visual summary of these five categories and typical stakeholders of the various sectors. These contexts are in some areas overlapping and interrelated. The last view, Energy/Electric Generation, Transmission \& Distribution, has the most obvious standards efforts related to GridWise. However, the technologies and standards efforts identified in the other views are not less important because in many cases they represent vast sectors of the economy, large sunk costs, or visionary new technologies that will be vital to the future of GridWise. ${ }^{7}$

In these separate sectors, different standards have emerged reflecting different business contexts, different technology contexts, and different semantic contexts. Current trends show that as technologies evolve and mature, and business practices overlap, standards efforts are now often focusing on convergence and interoperability. Global efforts toward convergence and interoperability in communications, information and business transaction technologies are as important to GridWise as are specific standards efforts in energy system control and communication.

\footnotetext{
${ }^{6}$ GridWise technical directions must also take into consideration regulatory and policy factors, but those factors are not within the scope of this report, so they are not specifically reflected in the viewpoints analyzed here.

${ }^{7}$ Figure 2-1 does not provide an exhaustive view of GridWise stakeholder groups, only those groups involved in standards development. Other stakeholders, such as regulatory and policy groups, are not directly addressed in this report.
} 


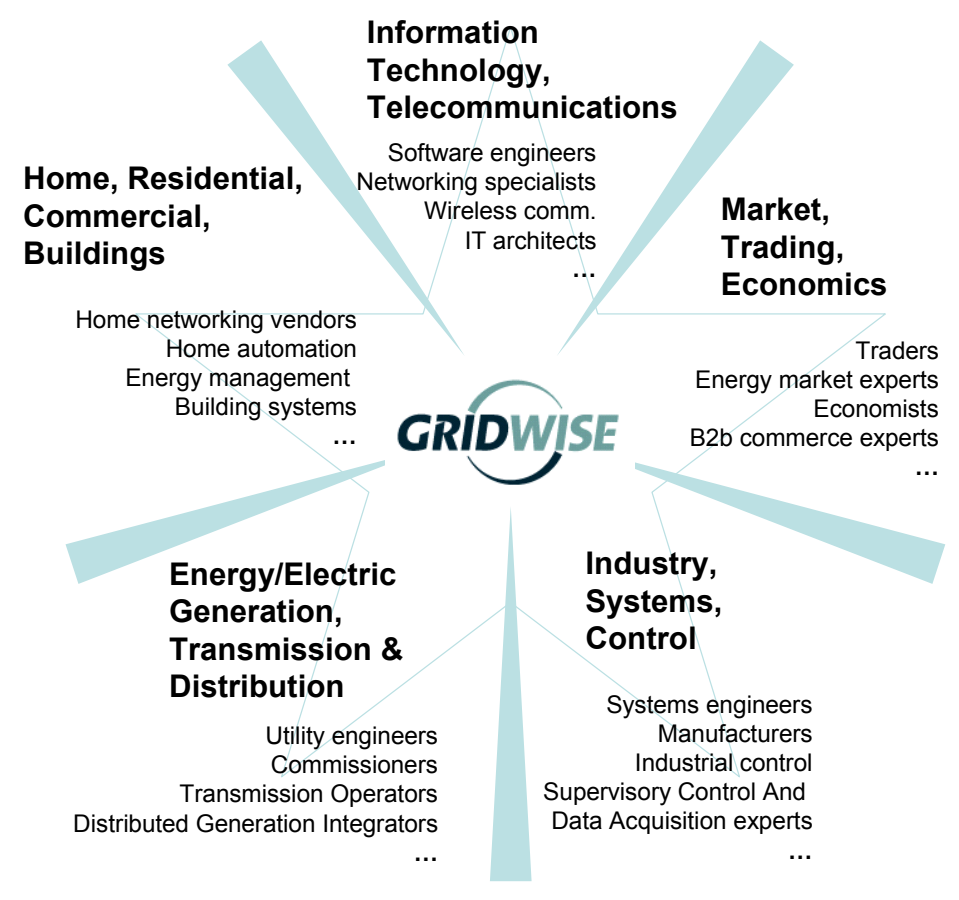

Figure 2-1 Dividing GridWise stakeholders into five general groups

The following tables summarize and categorize qualitatively the organizations and key standards efforts identified in this study:

- Table 2-1 Residential/Commercial/Buildings-Centered View of GridWise-Related Standards Efforts

- Table 2-2 IT and Telecommunications-Centered View of GridWise-Related Standards Efforts

- Table 2-3 Market/Trading/Economic-Centered View of GridWise-Related Standards Efforts

- Table 2-4 Industry/Systems/Control/Manufacturing-Centered View of GridWiseRelated Standards Efforts

- Table 2-5 Energy-Centered View of GridWise-Related Standards Efforts

\footnotetext{
${ }^{8}$ Further explanations and details of the relevant efforts of each group are provided in Appendix A.
} 


\section{A Home/Residential/Commercial/Buildings View}

Table 2-1 Residential/Commercial/Buildings-Centered View of GridWise-Related Standards Efforts ${ }^{9}$

Architecture/Guidance/Frameworks/Meta-views

AHAM-ARC-Appliance Technology Roadmap

OSGi-Architecture Expert Group

FIATECH--Capital Projects Technology Roadmap, Data Standards and Open Systems Resource

Management Aspects

IEEE-IAS - (Energy Systems Committee) P739 Bronze Book - Energy Management in Industrial and Commercial Facilities

Transverse Aspects (security, transactions, interoperability...)

ISO/IECJTC 1/SC 25/WG 1: Home Electronic Systems (Home Gateway...)

OSGi-Core Platform Expert Group (Service Gateway)

NIST-BRFL-BED-Mechanical Systems \& Control Group-BACnet

NIST-BRFL-BED-Computer-Integrated Building Processes Group

FIATECH--AEX - Automated Equipment Information Exchange

Systems, Integration, Models

CEA R7.4 Versatile Home Network Subcommittee-VHN (EIA-851-1394-Firewire-based)

CEA R7.2 Generic Common Application Language - CAL (EIA/CEA 721)

AHAM-Smart Appliance Task Force: Connected Home Appliances-Object Modeling (ANSI/AHAM CHA$1-2003)$

ISO TC 205 Building Environment Design WG 3 Building control systems design (internationalization of BACnet efforts--ISO 16484)

ASHRAE SPC 135: BACnet - A Data Communication Protocol for Building Automation and Control Networks (ISO 16484-5)

ASHRAE GPC-20: XML Definitions for HVAC\&R

Specific Applications

Home networks ${ }^{10}$ (networking protocols and communication media)

CEA R7.1 Home Controls Subcommittee-EIA/CEA-709.1 Control Network Protocol (LonWorks)

CEA R7.2 CEBus - Consumer Electronic BUS (EIA/CEA 600)

NIST-BRFL-BED-Mechanical Systems \& Control Group - Cybernetic Buildings

IEEE-Computer Society-Microprocessor Standard Committee --1394 series (FireWire)

Building \& Home (building control, energy management systems...)

NIST-BRFL-BED-HVAC\&R Equipment and Performance Group

ASHRAE SPC 111: Practices for Measurement...Balancing of Building HVAC and R Systems

ASHRAE Section 1.0 Fundamentals and General Technical Committees $1.4 \& 1.5$ (Control Theory and Application \&Computer Applications)

ASHRAE Section 7.0 Building Performance Technical Committees 7.4, 7.5 \& 7.6 (Building Operation Dynamics, Smart Building Systems \& Systems Energy Utilization)

\footnotetext{
${ }^{9}$ Other 'standards' that were not included within the scope of this study, but may be relevant include: Control \& Communications Protocols: Konnex EIB (European counterpart to BACnet); gateways and interoperability: UPnP, Home PnP; various network communication physical media: Bluetooth, IEEE 802.15 and other WiFi series, Home RF (SWAP), Home PNA/HomeRun.

${ }^{10}$ The X10 protocol and system (i.e., owned by X10 Ltd., often distributed by RadioShack, power-line carrier, very low data transfer rate) does not appear here because it is proprietary and has not been developed or published by an SDO.
} 


\section{An IT/Telecommunications View}

Table 2-2 IT and Telecommunications-Centered View of GridWise-Related Standards Efforts

Architecture/Guidance/Frameworks/Meta-views

IETF--IAB - Internet Architecture Board

ISO/IEC JTC 1/SC 7: Software Engineering

ISO/IEC JTC 1/SC 7/WG19: Modeling languages, metadata, ODP framework and components

ISO/IEC JTC 1/SC 7/SWG5: Architecture Management

NIST-MEL-MSID-eBSC, eBusiness Standards Convergence

OMG--Architecture Board

W3C--Technology and Society Domain: Semantic Web \& OWL (Web Ontology) Working Group

DISA--ASC X12C Communications and Control-TG03 EDI-Architecture

OASIS--ebXML Committees--Business Process, Collaboration, Interoperability, Registry...

Transverse Aspects (security, transactions, interoperability...)

UN/CEFACT--eBusiness Working Group: Architecture Specification

UN/CEFACT--UN/EDIFACT

ISO/IEC JTC 1/SC 6: Telecommunications and Information Exchange Between Systems

ISO/IEC JTC 1/SC 32/WG1: Business Agreement Semantic Descriptive Techniques (OPEN EDI)

ISO/IEC JTC 1/SC 7/WG9: Systems Integrity

IEEE-Computer Society-Task Force on Electronic Commerce

INCITS H2 - Technical Committee on Database

NIST-ITL-ANTD-Networking for Pervasive Computing Group

NIST-ITL-CSD-Cryptographic Standards and Applications: Public Key Infrastructure

NIST-ITL-SDCTD-Interoperability Group

ATIS-ASC T1, T1E1-- Interfaces, Power \& Protection for Networks

ATIS-ASC T1, T1M1.5-OAM\&P Architecture, Interface and Protocols

OMG Platform Technology Committee (UML, MDA, CORBA, CWM, XMI)

IETF--Security area: Securely Available Credentials (sacred)

IETF--Security area-- Secure Network Time Protocol (stime)

IETF--Security area--XML Digital Signatures (xmldsig)

OASIS--UDDI (Universal Description, Discovery and Integration) Web services registry

OASIS--Web Services Technical Committees

OASIS--Public Key Infrastructure TC

Business Processes and Management Aspects

ATIS-TCIF Electronic Ordering, EDI Service Order Committee, EDI Telecommunications Billing

OMG--Domain Technology Committee: ManTIS plus other domains

OASIS--UBL (Universal Business Language) Committees

Specific Telecommunications Applications

IETF--Application area: Electronic Data Interchange-Internet Integration (ediint)

ISO/IEC JTC 1/SC 6/WG7: Network, Transport, Directory, And ASN.1

IETF--Internet area: Zero Configuration Networking (zeroconf)

IETF--Internet area: Securing Neighbor Discovery (send)

ISO/IEC JTC 1/SC 6/WG1: Physical and Data Link Layers

IEEE-PES Power Systems Communications Committee, Power line carrier comm. systems.

IEEE-Computer Society-Technical Committee on Computer Communications (802 series)

IEEE-Computer Society-Microprocessor Standard Committee --1394 series (FireWire)

Specific IT Applications-GIS-- Testing and Testbeds

INCITS L1 - Geographic Information Systems

NIST-ITL-IAD-Pervasive Computing SmartSpace Testbed

NIST-ITL-SDCTD-Standards and Conformance Testing Group 


\section{A Market/Trading/Economics View}

Table 2-3 Market/Trading/Economic-Centered View of GridWise-Related Standards Efforts ${ }^{11}$ Architecture/Guidance/Frameworks/Meta-views

UN/CEFACT Business Collaboration Framework (BCF)

W3C--Architecture Domain: XML, Web Services, URI, DOM

Transverse Aspects (security, transactions, interoperability...)

RosettaNet--Universal Messaging Service

RosettaNet--Implementation Framework Core Specifications

ISO/TC 68/SC 2 - Security management and general banking operations (Framework for IT security for financial institutions, Public key infrastructure management for financial services)

IETF--Application area: Internet Open Trading Protocol (trade)

UN/CEFACT--International Trade and Business Process Group (TBG), UMM

UN/CEFACT--Techniques and Methodologies Group (TMG)

OASIS--Web Services Technical Committees

Management Aspects

UN/CEFACT--Business Process Working Group: Business Process Specification Schema

DISA--ASC X12F Finance Subcommittee, Energy and Utilities Task Group

Specific Applications

Software Agents

No open standards identified at this stage.

Financial Trading Exchanges

ISO/TC 68/SC 4 - Securities and related financial instruments (ISO 15022-- SWIFT, EDIFACT syntax, integrates XML)

\begin{tabular}{|l|}
\hline \multicolumn{1}{|c|}{ Auction Systems } \\
\hline No open standards identified at this stage \\
\hline \multicolumn{1}{|c|}{ Business Processes } \\
\hline $\begin{array}{l}\text { RosettaNet--Partner Interface Processes }{ }^{\circledR}(\text { PIPs } \\
\text { RosettaNet--Business Dictionary (RNBD) } \\
\text { RosettaNet--Global Company Identifier Program }\end{array}$ \\
\hline
\end{tabular}

This area is generally market-driven rather than standards-driven, but the trend toward crosscompany collaboration (XRP — extended resource planning) may drive further standardization in the future.

\footnotetext{
11 There are significant protocols used in the financial sector published by trade groups which were not in the scope of this study, including FIX protocol www.fixprotocol.org, and projects underway by the Financial Services Technology Consortium http://www.fstc.org/ (for example the UVX project, http://www.fstc-uvx.org).
}

There are also numerous implemented applications for financial exchange and auctioning, which often draw from underlying standardized technologies, but are not necessarily part of any standards process at this stage. Examples of these applications include: VerticalNet, FreeMarket, Ariba (trader and TradingDynamics), OpenSite, Moai, WellBid, AsAuction.con, AltraEnergy, Energy Window, agent-based work in academia, Global Trading Web, WebEx, CommerceOne, Primex, Silicon Summit Technology, OpenExchange On-line Bidding Software - OLB, LiveExchange (Intel), EIDX ITX, European EDIFICE Organization, Open Network for Commerce Exchange (ONCE-http://www.connect-once.com ). 


\section{An Industry/Systems/Control View}

Table 2-4 Industry/Systems/Control/Manufacturing-Centered View of GridWise-Related Standards Efforts

Architecture/Guidance/Frameworks/Meta-views

ISO TC 184/SC 5/WG 1-Enterprise modeling and architecture (ISO 14258, 15704, 19439, 19440)

ISO TC 184/SC 5/WG 5-Open systems application frameworks (ISO 15745)

NIST-MEL-ISD-RCS-Real-Time Control Systems Architecture for Intelligent Systems

ISO184 SC4-Standards for Industrial Data

Transverse Aspects (security, transactions, interoperability...)

NIST-MEL-MSID-Manufacturing Enterprise Integration

NIST-MEL-ISD-Open Architecture Control

OMG--Data Access Facility (DAF), Data Acquisition for Industrial Systems (DAIS), and Historical Data

Access from Industrial Systems (HDAIS)

ISO TC 184/SC 5/WG 6-Application service interface

OPC Security

OPC Data eXchange (OPC DX)

OPC Historical Data Access

OPC XML-DA: (OPC Data Access Specifications by using XML, supporting Web Services)

OPC Complex Data

ISA-SPTAG65, Industrial-Process Measurement and Control (USTAG for IEC TC65)

ISA-SP99, Manufacturing and Control Systems Security

ISA-SP72, Industrial Computer Interfaces and Data Transmission Techniques (MMS)

ASHRAE SPC 135: BACnet - A Data Communication Protocol for Building Automation and Control Networks (ISO 16484-5)

Management Aspects

IEEE-IAS-(Power Systems Engineering Committee) Industrial and Commercial Power Systems

Department (handles most color books)

IEEE-IAS - (Energy Systems Committee) P739 Bronze Book - Energy Management in Industrial and Commercial Facilities

Specific Applications ${ }^{12}$

\section{Manufacturing Control Systems}

ISO TC 184/SC 5/JWG 15-Joint ISO/TC 184/SC 5 - IEC/SC 65A WG : Enterprise-control system integration (ISO 62264)

ISO TC 184/SC 5/WG 2-Communications and interconnections (ISO 13283)

ISO TC 184 Industrial Automation Systems, SC 5--Architecture, communications and integration frameworks (ISO9506 MMS)

ISA-SP95, Enterprise/Control Integration Committee (ANSI/ISA-95 series, basis for ISO 62264)

OPC Data Access (OPC DA)

OPC Batch (corresponding to the S88.01 Physical Model (pre-S95, ISO62264))

OPC Commands

ISA-SP88, Batch Control

Systems Engineering

ISA-SPTAG65A, System Aspects (USTAG for IEC SC65A).

${ }^{12}$ Other related interest and trade groups include: http://www.profibus.com/, http://www.fieldbus.org, http://www.fdt-jig.org. 


\section{An Energy/Electric Generation, Transmission \& Distribution View}

Table 2-5 Energy-Centered View of GridWise-Related Standards Efforts

Architecture/Guidance/Frameworks/Meta-views

IEC TC 8: Systems aspects for electrical energy supply

IEC TC57/WG 16: Deregulated energy market communications

IEC TC57/SPAG: Strategic Policy Advisory Group

Business Aspects

NERC Functional Model Review Task Group

NAESB REQ Customer Process Subcommittee and Supplier-Utility Interface Subcommittee

NAESB WEQ Market Operations Subcommittee and Market Standards Subcommittee \& Contracts

Subcommittee

DISA--ASC X12F Finance Subcommittee, Energy and Utilities Task Group

Transverse Aspects (security, transactions, interoperability ...)

IEC TC57/WG 15: Data and communication security

IEEE-PES Power Quality Committee and Power System Dynamic Performance

NAESB WEQ: Electronic Scheduling Subcommittee

NERC Critical Infrastructure Protection Advisory Group, Public Key Infrastructure Steering Committee

NERC Interchange Subcommittee

NERC Data Exchange Working Group \& Telecommunication Working Group

Management Aspects

IEC TC57/WG 13: Energy Management System Application Program Interface

IEC TC57/WG 14: System Interfaces for Distribution Management Systems

Specific Applications

Components $\quad$ IEC TC22/SC22F: Power electronics for electrical transmission and distribution systems

IEEE-PES Power System Relaying Committee

IEEE-PES Transmission \& Distribution Committee

IEEE Power Electronics Society Standards Committee

Substations $\quad$ IEC TC57/WG 10-12: Communication standards for substations: Functional architecture and general requirements, Communications within and between unit and station levels, \& Communication within and between process and unit level

IEEE-SA SCC36: Utility Communications (UCA), IEEE TR1550 series.

IEEE-PES: Substations Committee, Data acquisition, processing, and control systems subcommittee (P1525, P1615, PC37.1, 1379...)

\begin{tabular}{l|l} 
Control & IEC TC57/WG 7 and WG 13: Telecontrol Protocols (TASE.2-ICCP)
\end{tabular}

Centers

Wholesale $\quad$ NAESB WEQ: Information Technology Subcommittee (OASIS-SCP standards)

DER $\quad$ IEC TC82: Solar PV, IEC TC88: Wind Turbines, IEC TC105:- Fuel Cells

IEEE-SA SCC21: 1547 series - Standard and Draft Standards for Interconnecting

Distributed Resources with Electric Power Systems

IEC TC57: new Working Group based on WG 10,11,12.

Generation $\quad$ IEEE-PES Energy Development and Power Generation Committee

Metering $\quad$ IEC TC13/WG 15: Electricity metering - Payment systems

and Retail IEC TC13/WG 14: Data exchange for meter reading, tariff and load control

IEEE-SA SCC31: Automatic Meter Reading and Energy Management

NAESB REQ Technical Electronic Implementation Subcommittee 


\subsection{Results from Views of GridWise-Related Standards}

The five viewpoints presented above-Home/Residential/Commercial/Buildings, IT/Telecommunications sectors, Market/Trading/Economics, Industry/Systems/Control, and Energy/Electric Generation, Transmission \& Distribution - provide a relatively clear set of windows into various facets of the vast world of standards and technologies related to GridWise. There are overlaps, and experts could easily debate the choices of categories and groupings chosen, yet these five viewpoints do allow us to arrange similar efforts together and gather some clear insight to the key issues in each domain.

This study has focused on standards groups and efforts that seem to have direct significance within the future scope of the GridWise vision-so only a fragment of the overall standards work of many of these groups is compiled here. The extent and range of different standards efforts in each of these various viewpoints remind us that GridWise interoperability must extend its vision well beyond the traditional frontiers of power and utility sector standards.

From the Home/Residential /Commercial/Buildings point of view, one might say GridWise technologies and standards efforts revolve around smart homes, smart appliances, smart buildings and smart communications. At the communication level for data transfer and transport, many standards efforts are considering different physical (wires or wireless) media and protocols for inhome or in-building data transfer, contending with issues such as existing or non-existing cabling and divergent needs between control and entertainment. At the data use level, efforts generally focus on building performance, management and dynamics or on home electronic systems and gateways between the home and external systems and services.

From the IT/Telecommunications point of view, many efforts are aimed at a higher level of frameworking and architecting. Within more specific efforts, we may note that there are extensive, different efforts underway surrounding e-business and security in transactions, as well as a visible effort toward interoperability.

The relatively few standards development efforts in the Market/Trading/Economics view point demonstrate the immaturity and market-driven tendencies in this area, where many implementations exist, but are not standardized, and much of the technological basis stems from academia. A considerable basis of rules and experience surrounding open market structures may be found in Security Exchange Commission rulings for markets such as Nasdeq, although the process and structure behind these rules does not follow a typical path of standards development. Though no specific SDO involved in these efforts was identified within the scope of this study, the Nasdeq example may provide valuable input for defining GridWise technical direction.

The Industry/Systems/Control viewpoint presents several standards areas that are historically linked to energy standards. It also demonstrates a tendency to move toward integrating XML compatibility into the world of industrial standards.

The Energy/Electric Generation, Transmission \& Distribution view point reflects the breadth of the energy sector, and complexity of communication and control technology and business process issues within this domain. Interestingly, there is only a very small number of SDOs involved in these standards, which may reflect the non-market-based past of these areas.

Within these viewpoints, the various efforts are also classified according to an approximate level of specificity - whether they address overarching issues such as architecture, transverse issues such as security, or specific applications. This dichotomy is not clear cut, but it does provide some structure for readers who are unlikely to be familiar with every viewpoint covered. 


\section{Multi-Dimensional Conceptualizations of this Standards Environment}

As presented above, the world of GridWise-related standards is complex, and mapping it poses great challenges. In some cases, different standards emanate from different SDOs covering similar scopes because they represent different regional directions (requirements and technological choices), and in some cases because they cover different niches or domains, or differing historical contexts. Even for standards organizations, it is difficult to draw the line where standardization stops to leave room for vendor or user tailoring of applications.

To provide further insight into the comparative positions of these numerous standards, this section plots some of the key efforts along various qualitative axes:

- Positioning with respect to the electric power sector (generation, transmission, distribution, end-use...).

- Horizontal positioning through various business sectors.

- Specificity or maturity of the standards work or technologies (does it address an abstract frame-work, guidance, design, protocols, device specifications, testing, etc.).

We also suggest a series of functional levels surrounding communication and control technologies, which can be helpful to position respective standards efforts and understand the extent of maturity of various types of standards.

\subsection{Business Sectors and Specificity of Standards}

Figure 3-1, Mapping Groups vs. Business Sectors, positions the identified standards efforts from some of the larger SDOs according to business sectors and according to the level of specificity of their work. Many of the standards efforts overlap more than one sector.

Not all standards efforts identified in this study have been included in Figure 3-1, and it does not address the Finance/Economics/Markets aspects. Nevertheless, this type of positioning can help to provide a simplified visual comparison of various efforts.

As would be expected, this representation shows a great number of identified GridWise-related efforts in the utility area, quite a few in information technologies, and specific efforts in the other related business sectors. The North American Energy Standards Board (NAESB) efforts are squarely within the utility area, where Institute of Electrical and Electronics Engineers (IEEE) and International Electro-technical Commission (IEC) and National Institute of Standards and Technologies (NIST) come across as strong organizations for bridging the gap between the utility sector and others. Some groups are more specific to a given sector, such as the Consumer Electronics Association (CEA) and the American Society of Heating, Refrigerating and AirConditioning Engineers (ASHRAE) address the Buildings sector and the OLE for Process Control (OPC) and Instrumentation, Systems and Automation (ISA) address industry and manufacturing.

The general dispersion and complexity of this perspective reflect a similar level of dispersion of standards efforts. Identifying spheres of influence or clear leaders in given sectors is not a straight forward task, and simplifications of this picture may result in an inaccurate and misleading understanding of existing standards efforts. 


\section{Mapping Groups vs. Business Sectors}

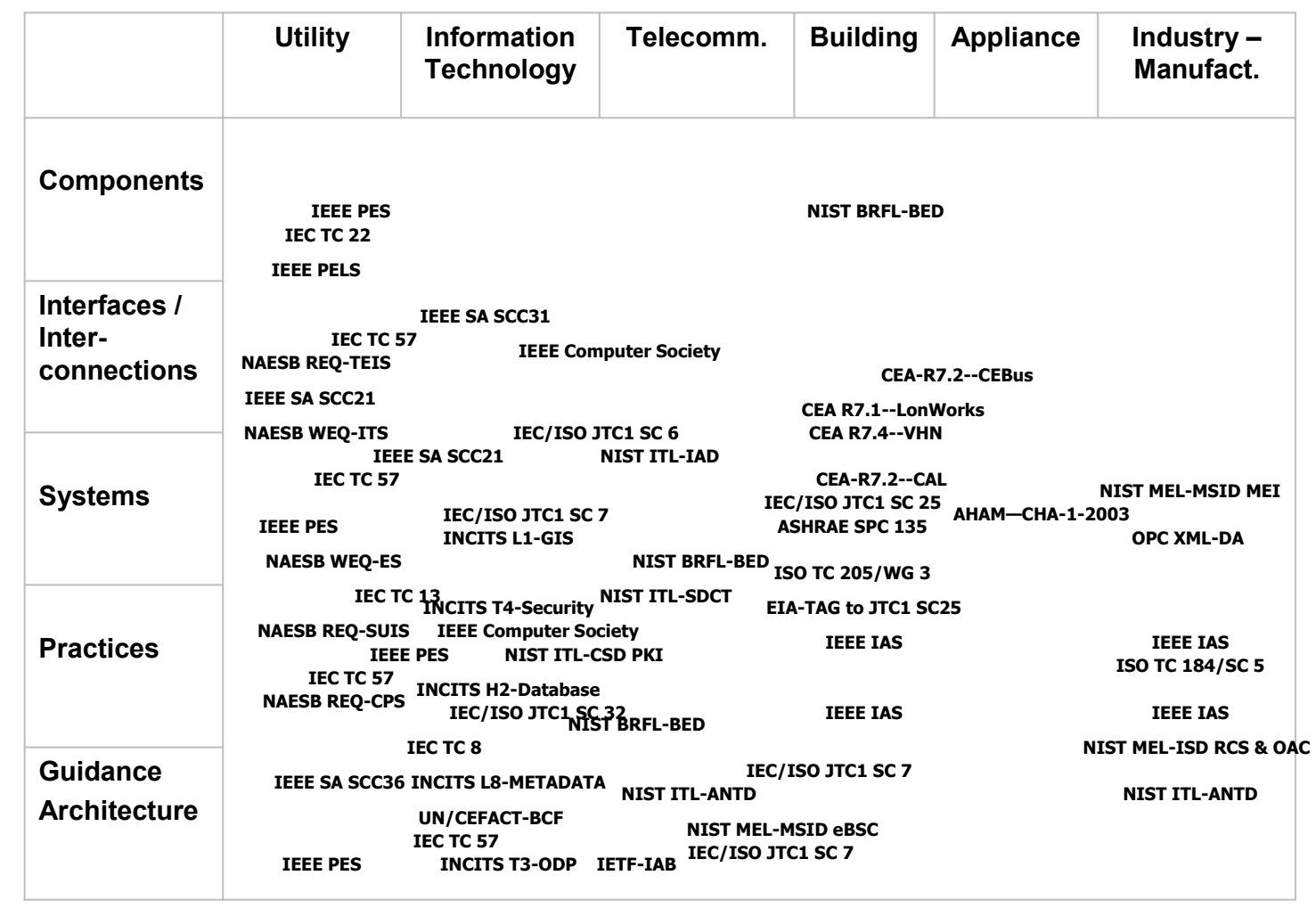

Figure 3-1 Mapping Groups vs. Business Sectors and Specificity. Many specific efforts overlap sectors, and many standards groups have efforts at different levels in several sectors.

\subsection{Mapping Standards Groups vs. Electric Sector Position}

Figure 3-2--Mapping Groups vs. Position provides a narrower mapping of these standards efforts, showing relative positioning and scope of NAESB, IEEE, and IEC GridWise-related efforts along the electric sector path. This view can help to visualize which efforts address more general, architectural issues, which are more specific in nature, and how various efforts are positioned within one domain. Most blatantly, however, it underlines the complexity and overlaps which reign. While just considering three of the many standards organizations related to GridWise, we can note that no single organization really addresses any one general sector, and all three organizations generate standards work at both higher abstract levels and at more specific levels.

The retail versus wholesale organization structure of NAESB reflects a focus on generation/transmission and a focus on distribution/end-use, but we see no bridging of the gap between the two areas and no overarching viewpoint encompassing the full spectrum of issues. The vertical lines associated with the various NAESB efforts indicate that within their specific sectors, each of these groups addresses standards at a range of levels, from business cases to terminology, or from design to specifications.

Similarly to NAESB, the IEEE does not have a unifying, guiding body that spans the various issues. Figure 3-2 shows that several efforts relevant to GridWise are underway within the 
different IEEE societies - the Power Engineering Society (PES), the Computer Society, the Instrumentation and Automation Society (IAS), and the Power Electronics Society (PELS) - and the various Standards Coordinating Committees (SCC) of the IEEE Standards Association. Together, these IEEE efforts span the different areas of the Electric Sector and address different levels of standards specificity and maturity.

The IEC standards groups relevant to GridWise typically address a relatively high standards level, providing guidance, architectural concepts and design level standards rather than focusing on specifications. IEC Technical Committee 8 (TC 8) is a recently formed committee that is likely to take a very high level systems-view. IEC TC 57 has several working groups at different levels, some of which focus on distribution aspects of the electric sector. The Joint Technical Committee 1 (JTC1) subcommittees cover areas related to information technology which may apply across the electric sector, with some which particularly address standards applicable to electric sector distribution and end-use. ${ }^{13}$

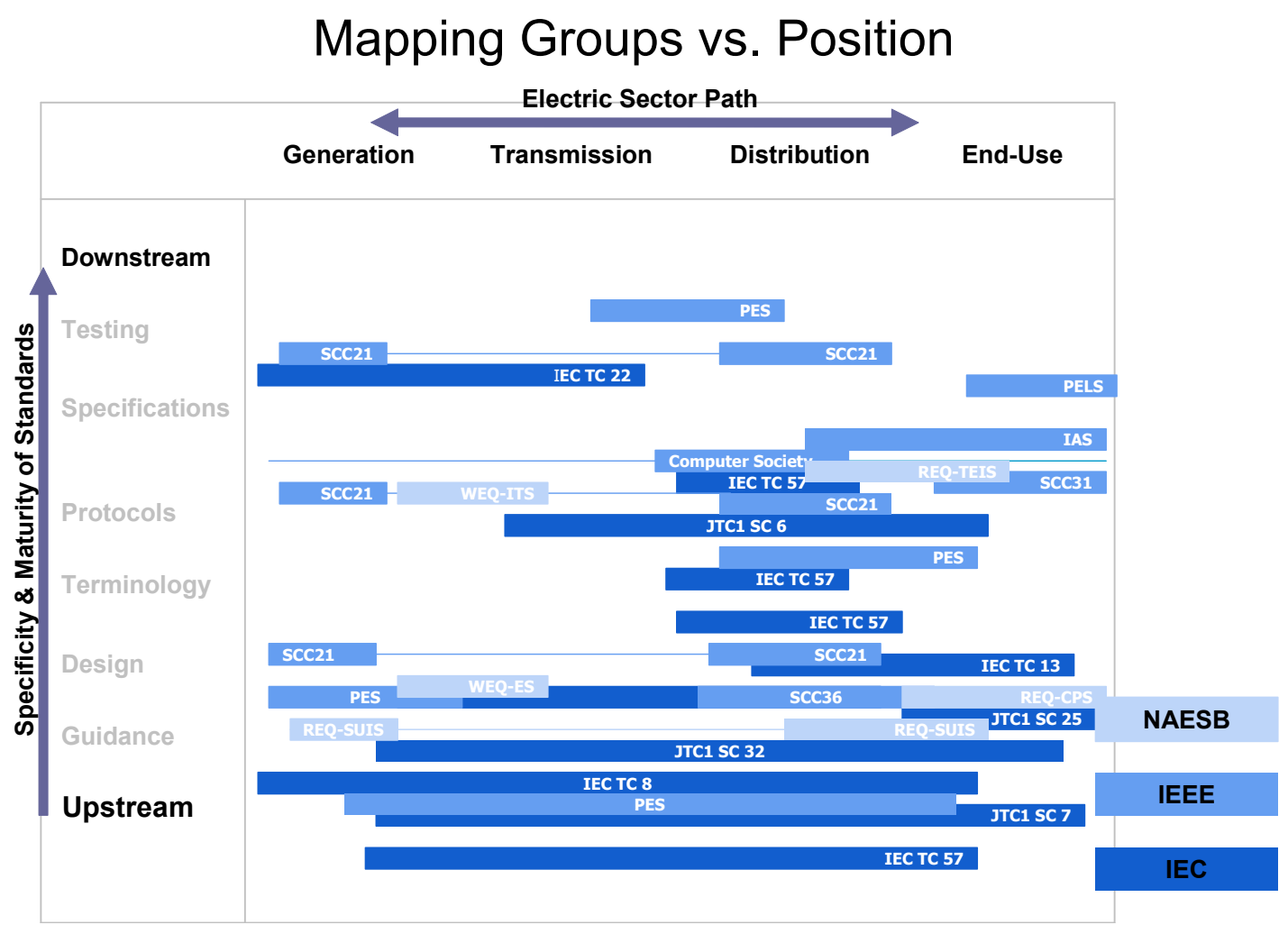

Figure 3-2 Mapping Groups vs. Position along Electric Sector Path. Reflecting the dispersion and organizational complexity which reigns.

\footnotetext{
${ }^{13}$ The Joint Technical Committee 1 (JTC 1) is the International Standards Organization/International Electro-technical Committee Joint Technical Committee for Information Technology (ISO/IEC JTC 1).
} 


\subsection{Functional Levels of Communication and Control Technologies}

Table 3-1: Functional Levels Surrounding Communication and Control Technologies on the following page details five different functional levels of technologies and standards that are relevant to GridWise. It presents examples and sub-layers of the various levels, and some comments to better explain the different levels. These five layers are summarized in Figure 3-3, below.

This schematic of functional levels surrounding communication and control technologies can help us understand and differentiate various standards related to GridWise. Some explanations of each level are provided below, to position each area in context historically and with respect to GridWise in a general sense. Transverse issues have not yet been addressed; these are domains that may affect several levels and are often treated separately by technical specialists and standards-making groups (interoperability, security, quality of service, conformance, etc.).

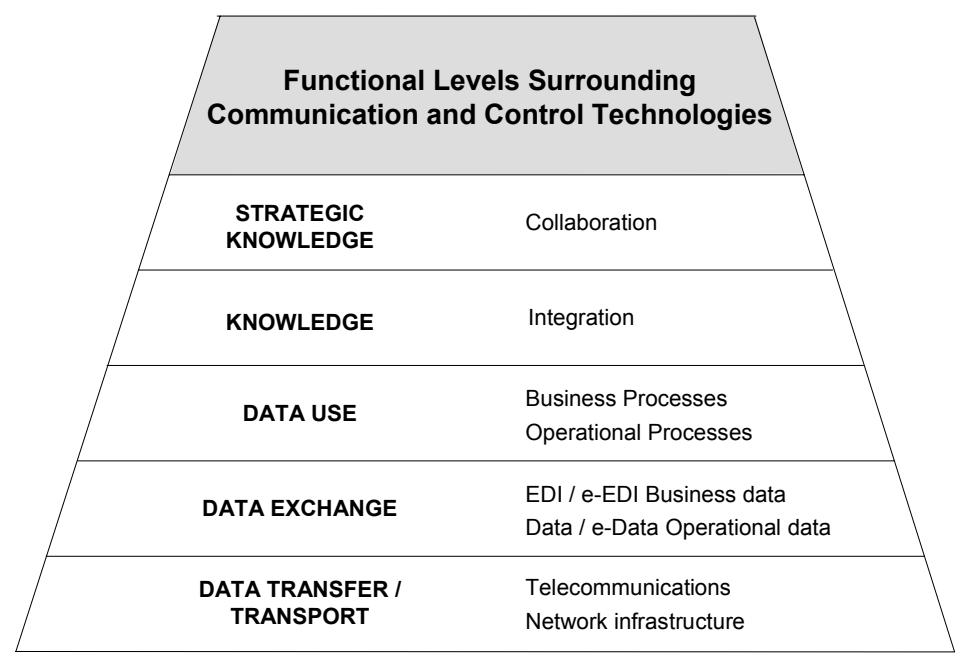

The data transfer/transport functional level

Figure 3-3 Summary of Functional Levels can be equated to the functions encompassed in the Open Systems Interconnection (OSI) sevenlayer model of network infrastructures (though some interpretations may put the top, application layer in one of the higher functional levels suggested here). ${ }^{14}$ Standards and technologies are quite mature in this layer, with relatively well established technologies (like TCP/IP and corresponding physical network media). New efforts are generally focusing on extending capacity (kilobits per second) for supporting applications such as home entertainment, or on reinforcing security and reliability (for example, the next generation of Internet Protocol, IPv6).

The data exchange level includes both transferring operational data and transferring business data. The contextual differences surrounding operational and business data result in different requirements (timeliness, authentication, volumes...). Specific standards exist now for electronic data interchange (EDI), and typically data exchange standards for operational data relies on domain specific standards. The convergence of many standards surrounding data integration, semantics, and syntax demonstrates the maturity of technologies and standards at this level. A large part of new standards in this level are extensions and new instances of existing data models and mappings.

\footnotetext{
${ }^{14}$ It is assumed readers are familiar with the ISO's Open Systems Interconnection (OSI) Architecture. Numerous works present this models, including Zimmerman 1980.
} 
Table 3-1 Functional Levels Surrounding Communication and Control Technologies

\begin{tabular}{|c|c|c|c|}
\hline Levels & Examples & Sub-layers & Comments \\
\hline $\begin{array}{c}\text { STRATEGIC } \\
\text { KNOWLEDGE } \\
\text { Data \& information re-use } \\
\text { across companies. }\end{array}$ & Collaboration & $\begin{array}{l}\text { Multi-function, multi- } \\
\text { company. }\end{array}$ & $\begin{array}{l}\text { XRP (eXtended Resource } \\
\text { Planning) } \\
\text { (Participants must choose } \\
\text { to contribute for mutual } \\
\text { advantages of } \\
\text { collaboration). }\end{array}$ \\
\hline $\begin{array}{c}\text { KNOWLEDGE } \\
\text { Data re-use for enterprise- } \\
\text { wide planning, decision- } \\
\text { making. }\end{array}$ & Integration & $\begin{array}{l}\text { Multi-function, one } \\
\text { 'roof' (company) }\end{array}$ & $\begin{array}{l}\text { ERP (Enterprise Resource } \\
\text { Planning) } \\
\text { Semantic web for } \\
\text { integration }\end{array}$ \\
\hline $\begin{array}{c}\text { DATA USE } \\
\text { In context, data content is } \\
\text { information, intelligent }\end{array}$ & $\begin{array}{l}\text { Business Processes } \\
\text { (using intelligent business } \\
\text { information) }\end{array}$ & $\begin{array}{l}\text { Transactions } \\
\text { Transaction Security }\end{array}$ & $\begin{array}{l}\text { Depends on context. } \\
\text { Uses and reacts to content. } \\
\begin{array}{l}\text { Depends on actors and } \\
\text { market factors }\end{array}\end{array}$ \\
\hline $\begin{array}{l}\text {--decision making } \\
\text {--triggering action/response }\end{array}$ & $\begin{array}{l}\text { Operational Processes } \\
\text { (using intelligent } \\
\text { operational information) }\end{array}$ & $\begin{array}{l}\text { Control } \\
\text { Monitoring } \\
\text { System Security }\end{array}$ & $\begin{array}{l}\text { Depends on context. } \\
\text { Uses and reacts to content. }\end{array}$ \\
\hline \multirow{2}{*}{$\begin{array}{l}\text { DATA EXCHANGE } \\
\text { Content of transferred } \\
\text { elements are structured, } \\
\text { codified, mapped, } \\
\text { integrated into another } \\
\text { system. } \\
\text { (Front-end/Back-end } \\
\text { Integration) }\end{array}$} & $\begin{array}{l}\text { EDI / e-EDI } \\
\text { (transferring intelligent } \\
\text { business information, } \\
\text { System A to System B) }\end{array}$ & $\begin{array}{l}\text { Integration } \\
\text { Semantics } \\
\text { Syntax }\end{array}$ & $\begin{array}{l}\text { EDI implies data transfer } \\
\text { between two separate } \\
\text { companies. } \\
\text { (Mapping, translating, } \\
\text { middleware, links, APIs) }\end{array}$ \\
\hline & $\begin{array}{l}\text { Data / e-Data } \\
\text { (transferring intelligent } \\
\text { operational information, } \\
\text { System A or Component A } \\
\text { to System B or Component } \\
\text { B) }\end{array}$ & $\begin{array}{l}\text { Integration } \\
\text { Semantics } \\
\text { Syntax }\end{array}$ & $\begin{array}{l}\text { Data exchange } \\
\text { (sometimes also called } \\
\text { EDI) not necessarily } \\
\text { involving separate } \\
\text { companies. } \\
\text { (Mapping, translating, } \\
\text { middleware, links, APIs) }\end{array}$ \\
\hline $\begin{array}{l}\text { DATA TRANSFER / } \\
\text { TRANSPORT } \\
\text { Bits and bytes are } \\
\text { transferred from point A to } \\
\text { point B. (Messaging) }\end{array}$ & $\begin{array}{l}\text { Telecommunications } \\
\text { (eg OSI seven layers) } \\
\text { Networking infrastructure }\end{array}$ & $\begin{array}{l}\text { (Comm. Protocols) } \\
\text { Network Security } \\
\text { Application } \\
\text { Comm. Management } \\
\text { Network, Routing } \\
\text { Transport } \\
\text { Physical }\end{array}$ & $\begin{array}{l}\text { Content and context } \\
\text { independent (though } \\
\text { different domains have } \\
\text { different requirements for } \\
\text { speed, reliability, security, } \\
\text { and different physical } \\
\text { contexts). }\end{array}$ \\
\hline
\end{tabular}

Technologies at the data use level take the function one step higher, using exchanged data to complete transactions or to enable control systems. Not only is data collected, transported and exchanged, it is also used by an application to make decisions and cause reactions. This level is 
less mature than the lower two levels - a plethora of different standards for different needs and different domains exemplify this immaturity. This is currently among the most active and evolving levels, as growing markets drive new implementations and standardization. Standards convergence efforts (such as NIST's eBusiness Standards Convergence, eBSC) are starting to progress for data use functions.

Both the knowledge level (integrating data use) and the strategic knowledge level (integrating data use across diverse entities for mutual collaboration) are market driven. These functions are typically addressed through unique implementations or proprietary systems, in some case with propriety standards or those maintained by a trade group. To enable functions at these levels, some high-level standards and research are addressing relevant issues such as semantic integration and knowledge management. In the GridWise context, the strategic knowledge level might encompass areas surrounding wide area monitoring, planning, and control. The knowledge level might encompass integration of EMS (energy management system), DMS (distribution management system) and ERP (enterprise resource planning) type systems.

\subsection{Using Functional Levels to Understand and Compare Standards}

These functional levels can help with landscaping the vast world of communication and control standards. The first example below considers standards from different sectors that are focused on the same level. The second example considers standards within one sector and categorizes them according to the different functional levels they address.

Examining standards from different sectors but focused on the same level allows us to examine a sub-group of related standards and identify similarities and differences in these various standards. For example, one might examine the OPC Data Access (DA) and XML DA (eXtended Markup Language Data Access) specifications and identify other standards currently in use in the electric power and building systems sectors that perform similar functions and how they compare methodologically to OPC DA or OPC XML DA.

Answering these sorts of questions will enable better characterization and appreciation of the roles and strengths of key standards. For example, gaining insight into the sources of differences, whether from different objectives, different environments (requirements like speed, reliability, security...), different approaches to control paradigms, different approaches to communication, backward compatibility requirements (e.g., legacy systems), or advancing generations (e.g., integrating XML or not). Gaining this knowledge will facilitate steps toward interoperability and convergence.

Examining standards within one sector with respect to the functional levels they address can help to clarify the roles played by these standards, with an objective of clarifying the roles of various standards, their similarities, complementarities, differences in context, and differences in technical approaches. For example, in the home networking sector, OSGi, UPnP, EIA/CEA 709 (LonWorks) and EIA/CEA 600 CEBus Standard, and EIA/CEA 721 Generic Common Application Language each fulfill different functions with some overlaps. ${ }^{15}$ Identifying the differences, overlaps, and points of convergence between these various standards within one sector can help to categorize and characterize the realm of standards.

\footnotetext{
${ }^{15}$ Numerous other standards also exist in home networking; just these few are listed here for illustrative purposes.
} 


\section{Conclusions}

This study provides an extensive compilation of the standards efforts in the major standards development organizations relevant to GridWise architecture. This world of standards is a complex, moving, evolving target, so this study only represents one snap-shot-necessarily limited in scope. The concepts within the GridWise vision are quite new and very little existing research is available drawing all of these concepts together, so this study is a starting point and should serve as one initial building block contributing to GridWise architecture needs. ${ }^{16}$ To attain its full value, this material will need to be reviewed and debated by experts from each stakeholder group and experts with knowledge at each functional level being considered; and updated accordingly.

It is clear in compiling and organizing this extensive information on standards and technologies that multiple viewpoints and visualization tools are necessary and useful to present this information and provide insight on the overall picture of GridWise-related standards. Summarizing within viewpoints from various sectors-Home/Residential /Commercial/Buildings, IT/Telecommunications sectors, Market/Trading/Economics, Industry/Systems/Control, and Energy/Electric Generation, Transmission \& Distribution-allows the various standards efforts to be grouped logically and recognizes the importance of each of these viewpoints.

Mapping the standards efforts according to various criteria allows us to visualize the relative positioning of these standards efforts. Also, using conceptual tools, such as identifying the functional levels of communication and control technologies can provide a point of reference for better understanding the interactions of various standards.

\subsection{Uses for this Study}

\section{Use of results to support formation of the Architecture Board:}

The first and most immediate application for this study is as a resource to aid in forming the GridWise Architecture Board. This study has allowed us to identify some domains and standards groups that may have otherwise been overlooked in our search for Architecture Board candidates. When the GridWise Architecture Board Nominations Committee is drawn together to draw up a slate of nominees for the Board, knowledge of the viewpoints presented in this study will help to construct an overall balanced slate, and to better evaluate the value of specific experiences of individual candidates.

\section{Use of results to support GridWise stakeholder outreach:}

A deeper comprehension of potential GridWise stakeholder communities will help us formulate and execute strategies for effectively reaching and communicating with key stakeholders. Understanding the technologies and standards efforts related to GridWise will assist in prioritizing outreach activities including where, when and how to stimulate and cultivate relationships with key efforts (inbound and outbound communication). Also this will enable more effective use of existing resources (researchers and other experts interested in contributing to GridWise), e.g., who is active in these standards groups (from near or far)? Identifying who

\footnotetext{
${ }^{16}$ The industry survey performed by the Integrated Energy and Communication Systems Architecture (IECSA) project has been a valuable, complementary source for this study (Sanza and Adamiak 2002).
} 
may act as effective contacts may allow us to better communicate to these individuals so they can be GridWise ambassadors and start to open pathways to create an initial outreach web, as effectively as possible with the least expenditure. A clearer understanding of standards efforts will allow us to better design how our 'ambassadors' can then communicate outwardly about GridWise and how they can further enrich GridWise Architecture efforts by bringing information back toward GridWise.

\section{Use of results as a reference:}

This study represents an essential resource for the future Architecture Board-compiling an initial, relatively detailed view of the world of standards related to GridWise. For more specific questions, the accompanying detailed draft reports on each SDO (summarized in Appendix A) may also serve as a quick reference-avoiding time spent searching for answers and information on the Internet. Similarly, others working toward the GridWise vision may find that this is a useful reference.

\subsection{Next Steps in Standards Mapping}

This standards mapping can be extended and improved in numerous ways:

- Gather feedback from experts in the various domains to improve and broaden this study.

- Create a detailed and cross-referenced glossary and index.

- Create overlap bubbles from each viewpoint, showing how these various standards efforts are related to one another across viewpoints and functional levels. Targeted studies should compare standards from different sectors but focused on the same level and examine standards within specific sectors to clarify their roles, similarities, complementarities, and different approaches.

- Create an on-line version of this study for easy access and reference.

- Identify and illustrate migration paths for similar standards which have converged, are being converged, or might/should be converged.

- As the GridWise architecture is initiated, identify interoperability gaps, duplications of efforts, and areas where direction and guidance need clarifying.

- Characterize key standards more fully analyzing their respective functional objectives, methodology, 'openness' (generic, open, proprietary), etc.

- Create an extension to this mapping which incorporates certain standards and technologies from trade groups and proprietary sources. Key efforts should be highlighted and integrated with the viewpoints presented in this study. 


\section{$5 \quad$ References}

Madnick, S.E. 2001. The Misguided Silver Bullet: What XML Will and Will NOT Do to Help Information Integration, Working Paper \#111, [Online paper]. Available URL: http://ebusiness.mit.edu/research/papers/111\%20SMadnick\%20Silver\%20Bullet.pdf, Center for eBusiness@MIT, Cambridge, Massachusetts.

Sanza, P. and M. Adamiak. 2002. A Guide to Existing Communications, Information, and Systems Architecture Technologies, Task 2 Final Report Revision 2, [Online report]. Available URL: http://www.iecsa.org. Prepared for J Hughes (Project Manager - EPRI/E2I) and D. Von Dollen (Program Manager - EPRI/E2I) on behalf of E2I and CEIDS board members.

Widergren, S. and M. Bosquet. 2003. "GridWise Architecture Tenets \& Illustrations," from the GridWise Architecture Board Call for Candidates Package, PNNL-SA-39480, Pacific Northwest National Laboratory, Richland Washington [Unpublished].

Zimmerman, H. 1980. "OSI Reference Model—The ISO Model of Architecture for Open Systems Interconnection," IEEE Transactions on Communications, Vol. 28, No. 4, April 1980, pp. 425-432.

\section{Bibliography}

Barry, D. 2003. Web Services and Service-Oriented Architectures, Morgan Kaufmann Publishers, San Francisco, California.

Becker, D., H. Falk, J. Gillerman, S. Mauser, R. Podmore, and L. Schneberger. 2000. "StandardsBased Approach Integrates Utility Applications." IEEE Computer Applications in Power, Vol.: 13, Issue: 4 , Oct. 2000 , pp. $13-20$.

Bell, K. and C. Morgan. 2003. "Power system analysis: emerging issues for utilities." Power Engineer, Volume: 17 , Issue: 2 , April/May 2003, pp. 26 - 30. Institution of Electrical Engineers, London, UK.

Bembibre J. and C. Samitier. 2003. "Web Services, a New Approach to Substation Management." From CIGRE Colloquium Rio de Janeiro, September 2003, D2-A04. [Online paper]. Available URL: http://www.cigre-sc35.org/Brazil.htm.

Chaudhury, J.P. and A. Kuilboer. 2002. e-Business and e-Commerce Infrastructure, Technologies Supporting the e-Business Initiative. McGraw Hill, New York.

DG "Enterprises" of the European Commission. 2003. 'European SMART HOUSE standardization in the eEUROPE context." From CENELEC Smart House Workshop, July 2003. [Online report]. Available URL: www.cenelec.org/NR/rdonlyres/C64C4570-6BFC-42AB-A4E26BE48FA4F241/ 0/FinalReportSmartHouse2nd.pdf.

Gibb, B.K. and S. Damodaran. 2003. ebXML: Concepts and Application. Wiley Publishing Inc, Indianapolis, Indiana.

IEC/TR 62357;2003(E). 2003. Power System Control and Associated CommunicationsReference Architecture for Object Models, services and protocols. International Electrotechnical Commission, Geneva, Switzerland. 
Instrument Society of America. 1989. "A Reference Model For Computer Integrated Manufacturing (CIM): A Description from the Viewpoint of Industrial Automation." From CIM Reference Model Committee International Purdue Workshop on Industrial Computer Systems. Purdue Research Foundation, West Lafayette, Indiana.

International Center for Standards Research. 2003. ICSR Papers \& Presentations. [Online resources]. Available URL: http://www.standardsresearch.org/presentations/index.html .

Kotok, A. 2002a. "Utility Deregulation Requires Effective E-Business Standards." [Online whitepaper]. Available URL: http://www.disa.org/whitepaper.cfm.

Kotok, A. 2002b. "Report of FERC Conference on Electric Industry Data Standards." July 22, 2002. [Online article]. Available URL: http://www.disa.org/whitepaper.cfm.

Krechmer, K. 1998. "The Principles of Open Standards.” Standards Engineering, Vol. 50, No. 6, November/December 1998, pp. 1-6. Standards Engineering Society, Miami, Florida. Available URL: http://www.standardsresearch.org/presentations/index.html

Krechmer, K. 2000. “Market Driven Standardization: Everyone Can Win.” Standards Engineering, Vol. 52 No. 4, July/August 2000, pp.15 - 19. Standards Engineering Society, Miami, Fl. Available URL: http://www.standardsresearch.org/presentations/index.html

Krechmer, K. 2002. "Cathedrals, Libraries and Bazaars." In Proceedings of the 2002 ACM Symposium on Applied Computing, pp. 1053-1057. ACM Press, New York, New York.

Lowell, S.C. 1999. "The Yin and Yang of Standards Development." ASTM Standardization News, Vol. 27, No. 12, December 1999, pp. 30 - 35. ASTM, West Conshohocken, Pennsylvania.

Putman, J.R. 2001. Architecting with RM-ODP. Prentice Hall PTR, Upper Saddle River, New Jersey.

Schubert, H. and G. Wong. 2003. "Towards Seamless Communications in Substations." Power Engineer, Vol.: 17 , No. 4 , Aug.-Sept. 2003, pp. 20 - 23. IEE, London, UK.

Hoffman, T. 2003. "Intelligent Buildings: Are We Finally Enabled?" CABA Home \& Building Automation Quarterly, Summer 2003, pp. 38 - 42. Available URL: http:/www.caba.org/.

Wacks, K. 2002. "Progress Towards Networked Appliances." CABA Home \& Building Automation Quarterly, Autumn 2002, pp. 20 - 24. Available URL: http://www.caba.org/.

Wacks, K. 2003. "IP Everywhere?" CABA Home \& Building Automation Quarterly, Summer 2003, pp. 19 - 23. Available URL: http://www.caba.org/.

Amended and Restated Memorandum of Understanding for the North American Energy Standards Board, North American Electric Reliability Council and the ISO/RTO Council, April 2003, Available URL: http://www.naesb.org/ 


\subsection{Main Websites of Standards Developing Organizations Studied}

Note that at some of the following groups were studied briefly, but do not appear in the detailing in Appendix A, which has been limited to include only the efforts with more significance for GridWise.

AHAM, Association of Home Appliance Manufacturers, http://www.aham.org/applresh/applresh.cfm.

ANSI, American National Standards Institute, http://www.ansi.org.

ASHRAE, American Society of Heating, Refrigerating and Air-Conditioning Engineers, http://www.ashrae.org, http://www.bacnet.org/.

ATIS (ASC T1), Alliance for Telecommunications Industry Standards, http://www.atis.org/; http://www.t1.org/.

BICSI, Building Industry Consulting Service International, http://www.bicsi.org.

BSI, British Standards Institute, http://www.ceem.com/standards.asp.

CABA, Continental Automated Buildings Association, http://www.caba.org.

CANENA, Harmonization of Electrotechnical Standards of the Nations of the Americas, http://www.canena.org.

CEA, Consumer Electronics Association, http://www.ce.org/.

CEN, European Committee for Standardization, http://www.cenorm.be/.

CENELEC, European Committee for Electrotechnical Standardization, http://www.cenelec.org/.

CIGRE, International Council on Large Electric Systems, http://www.cigre.org/.

DISA (defense), Defense Information Systems Agency, http://www.disa.mil.

DISA (X12), Data Interchange Standards Association, Inc., http://www.x12.org/x12org/index.cfm.

EAN, International, European Article Numbering, http://www.ean-int.org.

ECMA, European Computer Manufacturers Association or European Association for Standardizing Information and Communication Systems, http://www.ecma.ch/.

EIA, Electronic Industries Alliance, http://www.eia.org.

ETSI, European Telecommunications Standards Institute, http://www.etsi.org/.

FIATECH, Fully Integrated and Automated Technology, http://www.fiatech.org/.

IEC, International Electrotechnical Commission, http://www.iec.ch/.

IEEE-SA, IEEE Standards Association, http://www.standards.ieee.org/, http://www.ieee.org/.

IESNA, Illuminating Engineering Society of North America, http://www.iesna.org/.

IETF, Internet Engineering Task Force, http://www.ietf.org/. 
IPC, Association Connecting Electronics Industries, http://www.ipc.org/.

ISA, Instrumentation, Systems \& Automation, http://www.isa.org/.

ISO, International Standards Organization, http://www.iso.org/.

ITI(INCITS) (prev. ASC X3), Information Technology Industry Council, http://www.incits.org/.

ITU, International Telecommunications Union, http://www.itu.org/.

NAESB, North American Energy Standards Board, http://www.naesb.org/.

NAHBRC, National Association of Home Builders Research Center, Inc., http://www.nahbrc.gov/.

NEMA, National Electrical Manufacturers Association, http://www.nema.org/.

NERC, North American Electric Reliability Council, http://www.nerc.com.

NETA, InterNational Electrical Testing Association, http://www.netaworld.org/.

NFPA, National Fire Protection Association, www.nfpa.org.

NIST, National Institute of Standards and Technologies, http://www.nist.gov/.

NRECA, National Rural Electric Cooperative Association, http://www.multispeak.org, http://www.nreca.org.

OAGi, Open Applications Group, http://www.openapplications.org/.

OASIS, Organization for the Advancement of Structured Information Standards, http://www.oasisopen.org/.

OMG, Object Management Group, http:/www.omg.org/.

OPC, OLE for Process Control, http://www.opcfoundation.org/

OSGi, Open Services Gateway Initiative, http://www.osgi.org.

RosettaNet, http://www.rosettanet.org.

The Open Group, http://www.opengroup.org.

TIA, Telecommunications Industry Association, http://www.tiaonline.org.

UCC , Uniform Code Council, Inc, http://www.uc-council.net.

UL, Underwriter's Laboratory, http://www.ul.com.

UN/CEFACT, United Nations Center for Trade Facilitation, http://www.unece.org/cefact/index.htm.

UIG, Utility Industry Group, www.uig.org.

W3C, World Wide Web Consortium, www.w3c.org. 


\section{Appendix A \\ Standards Organization Details}




\section{A. Appendix: Standards Organization Details}

The extended standards mapping for these organizations currently focuses on those that have been judged to be of at least average significance for GridWise (this is a qualitative judgment, and organizations can be added or removed from this list as more information is acquired, or as GridWise architecture needs evolve). Table A-1 below shows the status of detailed investigations (whether sub-groups have been studied and assessed, standards identified, and implications for GridWise set forth.)

\begin{tabular}{|c|c|c|c|}
\hline \multicolumn{4}{|c|}{ Table A-1 Detailed Surveys of SDOs } \\
\hline SDO ACRONYM & Accredited? & Qualitative Significance & Completion of detailed survey drafts \\
\hline IEC & Int'1 SDO & Key & $\checkmark$ (plus extra appendix) \\
\hline IEEE & ANSI & Key & $\checkmark$ (plus extra appendix) \\
\hline NAESB & ANSI & Key or Average + & $\checkmark$ (included in document with NERC also) \\
\hline NIST & ANSI & Key or Average + & $\checkmark$ \\
\hline OMG & $\mathrm{N}$ & Average + & $\checkmark$ (included in document with W3C) \\
\hline ISO & Int'1 SDO & Average + & $\checkmark$ \\
\hline ASHRAE & ANSI & Average + & $\checkmark$ \\
\hline RosettaNet & $\mathrm{N}$ & Average+ + & $\checkmark$ \\
\hline $\mathrm{UN} / \mathrm{CEFACT}$ & Int'l Org. & Average + & $\checkmark$ (included with OASIS and DISA-X12) \\
\hline IETF & $\mathrm{N}$ & Average+ + & $\checkmark$ \\
\hline W3C & $\mathrm{N}$ & Average & $\checkmark$ (included in document with OMG) \\
\hline OASIS & $\mathrm{N}$ & Average & $\checkmark$ (included with UN/CEFACT and DISA-X12) \\
\hline CEA & ANSI & Average & $\checkmark$ \\
\hline EIA & ANSI & Average & $\checkmark$ \\
\hline AHAM & ANSI & Average & $\checkmark$ \\
\hline OPC & $\mathrm{N}$ & Average & $\checkmark$ (included in document with ISA) \\
\hline ITI-INCITS & ANSI & Average & $\checkmark$ \\
\hline DISA (X12) & ANSI & Average & $\checkmark$ (included with OASIS and UN/CEFACT) \\
\hline ANSI & NSB & Average & $\checkmark$ 一no key subcommittees. \\
\hline FIATECH & $\mathrm{N}$ & Average- & $\checkmark$ \\
\hline ATIS (ASC T1) & ANSI & Average- & $\checkmark$ \\
\hline OSGi & $\mathrm{N}$ & Average- or Average & $\checkmark$ \\
\hline ISA & ANSI & Average- & $\checkmark$ (included in document with OPC) \\
\hline NERC & ANSI & Average- & $\checkmark$ (included in document NAESB document) \\
\hline TIA & ANSI & Average- & $\checkmark$ (included in EIA/CEA document) \\
\hline OAGi & $\mathrm{N}$ & Average & Not detailed at this stage \\
\hline CABA & $\mathrm{N}$ & Average- or Average & Not detailed at this stage \\
\hline IPC & ANSI & Average- & Not detailed at this stage \\
\hline ITU & Int'1 SDO & Average- & Not detailed at this stage \\
\hline NEMA & ANSI & Average- & Not detailed at this stage \\
\hline NETA & ANSI & Average- & Not detailed at this stage \\
\hline NRECA & $\mathrm{N}$ & Average- & Not detailed at this stage \\
\hline
\end{tabular}




\section{A.1. Summary of Significant Sub-organizations}

For each SDO of greater than average significance, a draft report has been written, including:

- An overview (one page identity sheet) of the SDO

- A summary of committees and other subgroups with additional details on groups of interest to GridWise and information on standards efforts of interest to GridWise

- A summary of relationship to GridWise and implications for GridWise

- Appendix with further details on the various efforts, possible contacts toward the organization (within PNNL or within the organization), and other significant project details.

The objective in these surveys has been to perform a rigorous, though rapid, investigation with enough breadth to try to ensure that we have not missed key efforts (related to GridWise), and with enough depth within key identified efforts to grasp the scope and significance of the work. Of course, with such an extensive effort, compromises have been made to obtain a maximum understanding in a short time, and we can expect these working documents to evolve and be completed more fully as GridWise architecture efforts progress. Draft reports include:

- Standards Organizations Details-IEC

- Standards Organizations Details-IEEE

- Standards Organizations Details-NAESB \& NERC

- Standards Organizations Details-NIST

- Standards Organizations Details-UN/CEFACT, OASIS \& DISA

- Standards Organizations Details-IETF

- Standards Organizations Details-OMG \& W3C

- Standards Organizations Details-RosettaNet

- Standards Organizations Details-EIA \& CEA \& TIA

- Standards Organizations Details-OSGi

- Standards Organizations Details-AHAM

- Standards Organizations Details-ASHRAE

- Standards Organizations Details-FIATECH

- Standards Organizations Details-ISO

- Standards Organizations Details-OPC \& ISA

- Standards Organizations Details-ITI-INCITS

- Standards Organizations Details-ATIS

- Standards Organizations Details-ANSI

The following sections summarize information from each of the above draft reports, presenting the most significant efforts underway for each key organization. This information is based on publicly available online resources and may contain inaccuracies though every effort has been made to be as thorough as possible with the resources available as of December 2003. 


\section{A.1.1. Standards Organizations Details-IEC}

The International Electrotechnical Commission (IEC) has numerous committees working on GridWiserelated topics. As GridWise concepts and efforts progress, the significance of various technical committees and working groups will evolve. For initial stages focused on overarching architectural understanding, the key groups may be IEC TC 8--Systems aspects for electrical energy supply and IEC TC57/SPAG-Strategic Policy Advisory Group, though many other groups summarized in this document may also hold great potential for the GridWise architecture efforts.

Valuable work is in progress in several IEC technical committees (for example, TC57/WG10-16), but is not available to the general public while still in draft stages. It would be useful for GridWise Architects or technical support to have access to this work, either through more or less formal liaisons with IEC TC participants or informally through participants on the working groups in question.

\section{Summary of most significant groups within IEC:}

- IEC TC 8: Systems aspects for electrical energy supply (newly formed). Preparation, coordination and cooperation between TC's (Technical Committees) dealing with electrical energy supply-reliability, Connection practices, Operation, Network responsibility, Metering, Data exchange and balancing, Communication, Charging mechanisms for use of public supply systems, Outsourcing of network related services, Characteristics of energy supply.

\section{- IEC TC13 Equipment for Electrical Energy Measurement and Load Control}

- IEC TC13/WG 14: Data exchange for meter reading, tariff and load control (62056 series). COSEM: COmpanion Specification for Energy Metering; DLMS: Distribution Line Message Specification; HDLC: High-level Data Link Control; OBIS: OBject Identification System. A tool (an open standard) intended to allow energy traders, utilities, meter operators, data management companies to obtain metering data to support trading in deregulated energy markets.

- IEC TC13/WG 15: Electricity metering - Payment systems (62055 series) has a certain number of existing, published standards for payment systems. Future work will cover establishing standards for complete payment systems. (Generic processes, Static payment meters for active energy, Standard Transfer Specification).

- IEC TC22/SC22F: Power electronics for electrical transmission and distribution systems (addresses control and monitoring in power electronic components).

\section{- IEC TC57: Power System Control and Associated Communications}

- IEC TC57/WG 3: Telecontrol Protocols (60870 series-Telecontrol Equipment and Systems) ICCPInter-Control Center Communication Protocol; TASE.2-- Telecontrol Application Service Element.2 (more used in Europe), RTUs or Substation systems, object models, application Services, protocols, other control centers.

- IEC TC57/WG 10: Communication standards for substations: Functional architecture and general requirements, communication standards for substations (61850 series-SAS, Substation devices (switchgears, transformers, protection, control, \& metering), IED Field Devices, Related Object Models, ACSI-- Abstract Communication Service Interface; equivalent to UCA Common Application Service Model (Get, Set, Reporting, Logging, Control, ...) CASM, Mapping to MMS-- Manufacturing Message Specification) Power quality monitoring, Conformance testing.

- IEC TC57/WG 11: Communication standards for substations: Communications within and between unit and station levels (61850 series) Communication networks and systems in substations, Specific communication service mapping (SCSM) - Mapping to MMS. 
- IEC TC57/WG 12: Communications standards for substations: Communication within and between process and unit level (61850 series) Specific communication system mappings (SCSM) - Sampled value.

- IEC TC57/WG 13: Energy Management System application program interface (EMS - API) (61970 series - Common Information Model, CIM; Component Interface Specification, CIS; Application Interfaces) Guidelines and general requirements, glossary, CIM base, financial, energy scheduling, reservations, CIS framework, Common data access facility, CIM XML Codification for Programmable Reference and Model Data Exchange, EMS-API Exchange of Graphics Schematics Definitions (Common Graphics Exchange). EMS \& SCADA.

○ IEC TC57/WG 14: System Interfaces for Distribution Management Systems--SIDMS (61968 seriesCIM for Enterprise Application Integration, EAI) System interfaces for distribution management, for network operations, Interface Standard for Records and Asset Management. Middleware Services compliancy specifications for distribution management business functions, plus EMS, plus retail, plus Supply Chain, plus ERP, etc.

- IEC TC57/WG 15: Data and communication security (recent). Work in this group may be based on IEC/TR 62210 (2003-05) -- Power system control and associated communications - Data and communication security.

- IEC TC57/WG 16: Deregulated energy market communications (recent). Work in this group will continue on 62195-Electronic communications in deregulated energy markets (2000). The current draft in progress is 62325 - Framework for Deregulated Electricity Market Communications. ${ }^{17}$

- IEC TC57/SPAG: Strategic Policy Advisory Group. The TC 57's Strategic Policy Advisory Group (SPAG) provides a platform for the coordination among the different working groups, ensuring that the communication architecture is backward compatible and includes migration strategies and paths for legacy protocols. As a first step towards this communication architecture SPAG is presently investigating the usage of IEC 61850 for communication not only within the substation but also between substation and control center.

- ISO/IEC JTC 1/SC 6 Telecommunications and Information Exchange Between Systems. Standardization in the field of telecommunications dealing with the exchange of information between open systems including system functions, procedures and parameters and equipment as well as the conditions for their use. This standardization includes both the lower layers that support the physical, data link, network and transport services, including private integrated services networking, as well as the upper layers that support the application protocols and services.

- ISO/IEC JTC 1/SC 6/WG1: (Telecommunications and information exchange between systems) Physical and Data Link Layers (Subjects 3,5,11)

- ISO/IEC JTC 1/SC 6/WG7: (Telecommunications and information exchange between systems) Network, Transport, Directory, And ASN.1 (30,31,32,33,34,35,40,46,47)

- ISO/IEC JTC 1/SC 7 Software Engineering. Systems view, frameworks, metadata.

○ ISO/IEC JTC 1/SC 7/WG9: Systems Integrity

○ ISO/IEC JTC 1/SC 7/WG19: Modeling languages, metadata, ODP framework and ODP components

- ISO/IEC JTC 1/SC 7/SWG5: Architecture Management

${ }^{17}$ IEC 62325 Ed. 1.0 CD (Committee Draft). 2003. Framework for Energy Market Communications. International Electrotechnical Commission, Geneva, Switzerland. 
- JTC 1/SC 25: Interconnection of Information Technology Equipment, ISO/IECJTC 1/SC 25/WG 1, Home Electronic Systems. Guidelines for product interoperability; a residential gateway model for home electronic system.

- ISO/IEC JTC 1/SC 32/WG1: (Data Management Services): Business Agreement Semantic Descriptive Techniques (OPEN EDI), working with EDIFACT and OASIS ebXML, both business and information perspectives for the needs of electronic commerce, electronic administration, electronic business.

- For specific DER resources, IEC has technical committees for specific technologies (TC82-Solar PV, TC88-Wind Turbines, TC105- Fuel Cells).

\section{A.1.2. Standards Organizations Details-IEEE}

The Institute of Electrical and Electronics Engineers Standards Association (IEEE-SA) and IEEE societies have numerous committees working on GridWise-related topics. As GridWise concepts and efforts progress, the significance of various technical committees and working groups will evolve. Because there is no architectural or integrative approach in IEEE groups, it is not obvious which particular group would be key for initial needs of the GridWise Architecture Board, although several specific efforts will be of great interest to GridWise (for example, SCC21 P1547).

GridWise architecture would benefit from additional information on current undertakings of SCC36 (and in general, a more accurate picture of GridWise-related efforts in IEEE), this information is not readily obtainable to non-members of committees.

This snapshot underlines the compartmentalization of GridWise-related efforts in the IEEE and the lack of any overarching, guiding mechanism between these numerous efforts.

Summary of most significant groups within IEEE and IEEE-SA:

- IEEE-SA SCC21: WG P1547.1, P1547.2, P1547.3 - Standard and Draft Standards for Interconnecting Distributed Resources with Electric Power Systems (Testing; Application Guide; Monitoring, Exchange and Control)

- IEEE-SA SCC31: Automatic Meter Reading and Energy Management (P1480) Standard for Application Layer (OSI Layer 7) Language Minimal Services and Parameters for the End-to-End Transport of Table Information in an Automatic Meter Reading Environment

- IEEE-SA SCC36: Utility Communications (UCA). Linked to the adoption of EPRI's UCA v.2.0 as IEEE Technical Report 1550. Currently working on adapting UCA to Gas Utility needs.

- IEEE-PES Power Quality Committee (recently established, P1409). Has several projects on power quality standards underway.

- IEEE-PES Substations Committee, Data acquisition, processing, and control systems subcommittee (P1525, P1615, PC37.1, 1379, etc.) Recommended practices for communication, control, and interoperability within substations.

- IEEE-PES Power Systems Communications Committee (need to discover what is active currently) Power line carrier communication systems.

- IEEE-PES Power System Dynamic Performance (no standards development), studies power system stability controls.

- IEEE-PES Power System Relaying Committee (active in P1525 and C37 along with PES-Substations Committee) addresses communications from point of view of specific components (protective relays and other IEDs). 
- IEEE-PES Transmission \& Distribution Committee (P1409?) Focus on T \& D components, previously addressed power quality, but seems to have transferred some topics to the Power Quality Committee.

- IEEE-PES Energy Development \& Power Generation Committee (1046-1991, and other generation-side standards) distributed digital control and monitoring for power plants.

- IEEE-IAS - (Power Systems Engineering Committee) Industrial and Commercial Power Systems Department (most color books) Fundamental references for best practices related to power systems.

- IEEE-IAS - (Energy Systems Committee) P739 Bronze Book - Energy Management in Industrial and Commercial Facilities (best practices).

- IEEE-Computer Society-Technical Committee on Computer Communications (802 series) Fundamental standards for LANs (subsections address different physical network media.)

- IEEE-Computer Society- Task Force on Electronic Commerce (no standards development, just participation)

- IEEE-Computer Society-Microprocessor Standard Committee 1394 series (FireWire) Plug and play type, real-time (isochronous) information sharing for home networks (printers, scanners, cameras...).

- IEEE Power Electronics Society Standards Committee (1573-2003) Recommended Practice for Electronic Power Subsystems: Parameters, Interfaces, Elements, and Performance

\section{A.1.3. Standards Organizations Details-NAESB \& NERC}

The draft report on the North American Energy Standards Board (NAESB) and the North American Electric Reliability Council (NERC) presents an overview of the relationship between NERC, NAESB and the Independent System Operator/Regional Transmission Organization Council (ISO/RTO) Council concerning standards and industry policy, as well as further details of key standards such as OASIS-SCP ${ }^{18}$ and e-tagging. Most of the higher level standards work is currently being transferred from NERC to NAESB, though significant 'reliability' aspects remain under NERC.

The Retail EQ is starting down a path toward more open standards development (combining $\mathrm{REQ} / \mathrm{RGQ} / \mathrm{WGQ}$ for ET efforts, and tying it to IETF work). Also, their approach seems to follow more modular tendencies as is typically seen in eCommerce software engineering.

The Wholesale EQ is tied into the very extensive OASIS-SCP system, which does not yet show tendencies to follow similar development paradigms to other eCommerce efforts. Their task is complicated by uncertainties in the market structure and regulatory directions, but it could be aided by modularizing their standards efforts such that various pieces of the puzzle can benefit from broader progress in eCommerce standards and software engineering.

Although the ISO/RTO Council signed the MOU, they do not develop standards. It is not clear where lines will be drawn from wholesale to retail, and where standards questions integrating transmission and distribution will be treated.

\footnotetext{
${ }^{18}$ Note that to avoid confusion between the NAESB/NERC OASIS system and the OASIS standards organism (ebXML initiator with the UN/CEFACT), the energy sector's OASIS system is referred to as OASIS-SCP (for Open Access Same-Time Information System-Standards and Communication Protocol) in this document.
} 


\section{Summary of most significant groups within NAESB and NERC:}

\section{NAESB REQ--Retail Electric Quadrant}

- NAESB REQ Customer Process Subcommittee: standards and model business practices (including data dictionaries and complementary documentation) involving customer processes, with an initial focus on (1) billing and payments, (2) customer enrollment and switching, (3) customer information and (4) customer inquiries.

- NAESB REQ Supplier-Utility Interface Subcommittee: standards and model business practices (including data dictionaries and complementary documentation) involving the working relationships between retail energy suppliers and the Local Distribution Company implementing retail access in its service area, focus is on (1) creditworthiness, (2) supplier licensing, (3) retail meter data validation, editing \& estimating, (4) load profiling, (5) market participant interactions, (6) utility-supplier disputes and (7) settlement process.

- NAESB REQ Technical Electronic Implementation Subcommittee: develop recommendations for transaction sets, data communication standards and implementation guidelines, the focus involves data communications, ANSI data element and transaction set definitions, particularly electronic delivery mechanisms associated with (1) billing and payments, (2) customer enrollment and switching, (3) metering, (4) load profiling and (5) customer information.

\section{WEQ: Wholesale Electric Quadrant}

- NAESB WEQ: Information Technology Subcommittee (newly formed, kick-off meeting was Oct 8, 2003, is to develop standards and enhancements to existing standards concerning technical implementation issues for OASIS-SCP 1A and OASIS-SCP II; and act as a 'home' for previous OSC members) (It is not clear whether it is this subcommittee or another technical subcommittee which is addressing the area of eMARC-PKI).

- NAESB WEQ: Electronic Scheduling Subcommittee: newly formed, not yet active, intended to continue ESC work on E-Tagging business standards (OASIS-SCP 1A and OASIS-SCP II issues of the ESC). It seems, though it is not yet certain, that the ES will be more oriented to business processes than the ITS.

- NAESB: Other Committees defining related business processes and contracts:

○ WEQ Market Operations Subcommittee

- WEQ Market Standards Subcommittee

○ WEQ Contracts Subcommittee

\section{NERC}

- NERC Operating Committee

- Functional Model Review Task Group: redefining the NERC Reliability Functional Model defining functions (roles), responsibilities, and interactions in the overall process of operating the wholesale/transmission system.

- Data Exchange Working Group \& Telecommunication Working Group: develops data management and transfer specifications for the Interregional Security Network, oversees planning, implementation, and use of the ISN , monitors functional performance of the ISN, and recommends changes to the ISN (data dictionaries, registries, methods...)

- Interchange Subcommittee: home to the Transaction Information System Working Group responsible for e-tagging. It is not clear which aspects of this work will be transferred to 
NAESB.

- NERC Critical Infrastructure Protection Advisory Group

- Public Key Infrastructure Steering Committee: designing and implementing a Public Key Infrastructure (PKI) for the electronic security of existing and future computer-based systems used to exchange data within the electric sector, encompassing privacy, authentication, integrity, non-Repudiation. These protections require the use of public key cryptography and public key certificates to bind a person's or computer system's public key to their identity and to support symmetric encryption key exchange.

\section{A.1.4. Standards Organizations Details-NIST}

The National Institute of Standards and Technology (NIST) has a long history of addressing issues of standards convergence and interoperability, as well as a solid record of cooperative, effective liaisons will industry and business sectors. NIST also has active participation and liaisons with key groups for GridWise in other SDOs (ISO, IEC, JTC1, IEEE, TIA, ITU-T, INCITS, IETF, EIA, ASHRAE, W3C, OASIS, OMG, FIATECH...).

\section{Summary of most significant groups within NIST:}

\section{Manufacturing Engineering Laboratory (MEL)}

- Manufacturing Systems Integration Division (MSID)

- Manufacturing Enterprise Integration (interoperability testbed for Semantic Web concepts)

- eBSC eBusiness Standards Convergence (multi-industry/sector forum investigating e-business standards convergence)

- Intelligent Systems Division (ISD)

- Open Architecture Control (interoperability of control systems for machines on the factory floor with design and planning systems, with factory data networks and with each other)

- RCS-Real-Time Control Systems Architecture for Intelligent Systems (an open and scalable Reference Model Architecture, suitable for many software-intensive, real-time control problem domains.)

\section{Information Technology Laboratory (ITL)}

- Advanced Network Technologies Division

- Networking for Pervasive Computing Group (characterizing behavior and performance of servicediscovery protocols, and develop an architectural specification that encompasses the general features provided by a range of commercial service-discovery protocols).

- Information Access Division

- Pervasive Computing SmartSpace Testbed (testbed for pervasive computing research efforts)

- Computer Security Division

- Cryptographic Standards and Applications: Public Key Infrastructure (numerous computer security standards areas). 
- Software Diagnostics and Conformance Testing

- Standards and Conformance Testing Group (conformance testing standards for primarily ebXML messaging technologies. including registry, business process, core components and messaging services)

- Interoperability Group

\section{Building and Fire Research Laboratory (BFRL)}

- Building Environment Division

- Mechanical Systems \& Control Group (Cybernetic building systems, communication systems and network security-BACnet...integrated ECMS).

- HVAC\&R Equipment and Performance Group (efficiency and reliability of HVAC\&R equipment)

- Computer-Integrated Building Processes Group (Standard Building Information Models, Interoperability Standards for Capital Facilities - Improving Equipment Design, Specification, Purchase, Fabrication, and Installation)

\section{Summary of most significant projects within NIST:}

- eBSC (eBusiness Standards Convergence Forum). Industry forum developing a framework and principles for developing and converging cross-industry standards for eBusiness capabilities and for achieving long-term interoperability.

- Pervasive Computing Program-Service Discovery Research. Extensive NIST service-discovery research project aimed at characterizing behavior and performance of service-discovery protocols, and develop an architectural specification that encompasses the general features provided by a range of commercial service-discovery protocols.

- Interoperability Standards for Capital Facilities. Assisting U.S. industries in developing and implementing interoperability standards for capital facilities (embodied in the FIATECH effort).

- $\quad$ Intelligent Open Architecture Control of Manufacturing Systems. Open architecture control, a common architecture of system components and interfaces, to realize the benefits of increased intelligence in manufacturing processes.

- Cybernetic building systems, communication systems and network security in building systems (Mechanical Systems \& Control Group, BACnet, integrated ECMS).

\section{A.1.5. Standards Organizations Details-UN/CEFACT, OASIS \& DISA}

The work of these three groups is interrelated and complementary, each focusing on different aspects of electronic commerce. Historically, the Data Interchange Standards Association (DISA) Accredited Standards Committee X12 maintained and developed EDI standards prevalent in legacy systems in the US. The United Nations Centre for Trade Facilitation and Electronic Business (UN/CEFACT) developed and maintained the EDIFACT standards (international EDI standards). ${ }^{19}$ With the joint ebXML initiative

\footnotetext{
${ }^{19} \mathrm{UN} / \mathrm{CEFACT}$ EDIFACT standards have a syntax somewhat similar to X12's EDI standards and they follow basically the same principles and architecture. Read 'Utility Deregulation Requires Effective eBusiness Standards', Kotok, Alan, DISA, June 2002 for further background.
} 
between the Organization for the Advancement of Structured Information Standards (OASIS) and the $\mathrm{UN} / \mathrm{CEFACT}$, all of these organizations began encompassing internet technologies and issues in their scopes and working toward standards which support 'open-edi'. Where 'open-edi' implies support for ebusiness using internet technologies and including support for existing EDI systems and migration toward more recent paradigms.

All three of these groups focus on business transactions as opposed to 'process' transactions (needed for real-time operations, for example). The UN/CEFACT focuses on business practices and processes and provides modeling methodology for open-edi business processes. OASIS continues to develop and maintain the ebXML framework and some of its associated standards (though many of the underlying standards specified within ebXML stem from other groups such as the IETF, W3C or the OMG). The ASC X12 group within DISA focuses on ensuring integration and migration of older EDI standards into the open-edi frameworks. DISA also works on specific development issues supporting and working actively with the UN/CEFACT and OASIS.

UN/CEFACT's recently announced Business Collaboration Framework promises to be a very high-level architecture allowing for interoperability of numerous technologies for business transactions. It aims to be an overarching, uniting framework enabling business process and information modeling.

\section{UN/CEFACT}

\section{Summary of most significant groups within UN/CEFACT:}

- International Trade and Business Process Group (TBG)-- responsible for the simplification of international trade procedures, business and governmental process analysis, and best practices, using the UN/CEFACT Modeling Methodology (UMM) where appropriate to support the development of trade facilitation and electronic business solutions.

- Numerous domain specific working groups (including among others transport, finance, architecture \& engineering).

- Techniques and Methodologies Group (TMG)-- to provide all UN/CEFACT Groups with Meta (base) Business Process, Information and Communications Technology specifications, recommendations and education. The TMG shall also function as a research group evaluating new information and communication technologies (ICT), as well as, techniques and methodologies that may assist UN/CEFACT and its groups to fulfill their mandate and vision in Trade Facilitation and e-Business.

- UN/CEFACT Business Collaboration Framework (BCF), a technological and implementation neutral approach to the exchange of global information requirements for governmental, commercial and industrial organizations. This is the UN/CEFACT's new 'flagship,' overarching architectural effort.

- eBusiness Working Group: Architecture Specification-- to produce the eBusiness Architecture and supporting documents, which include the ebXML architectural framework. This project will also ensure that the electronic business initiatives are technically and practically implementable and that the eBusiness architecture meets the requirements of business on a global scale. The scope of this project is to create a cohesive infrastructure for all electronic business formats including, but not limited to, ebXML, and EDI, by recognizing and developing emerging technologies for use within the umbrella of UN/CEFACT.

- Business Process Working Group: 1) Business Process Specification Schema-- to coordinate changes to the ebXML business process specification schema. This project will help ensure that Business Process Specification Schema and related specifications have alignment and support the ebXML vision of electronic commerce (BPSS 1.01). 2) Maintaining and improving UN/CEFACT Modeling Methodology (UMM) - meta- 
model, reference guide, user guide....

- UN/EDIFACT - the long-established international standards and specifications for electronic data exchange (has many similarities to ASC X12 EDI).

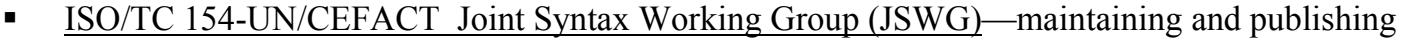
syntax specifications for EDIFACT.

On August 21, 2003 the UN/CEFACT announced the successful completion of the ebXML technical standards work program with OASIS after adoption of the ebXML specifications by the UN/CEFACT in May 2003. It is not clear at this stage how the relationship between UN/CEFACT and OASIS will evolve henceforth. The new 'flagship' initiative in e-business in the UN/CEFACT is their Business Collaboration Framework, presenting a high level architecture enabling business transactions (and allowing for a multitude of underlying technologies-EDI, UBL, Web Services.... $)^{20}$.

\section{OASIS}

\section{Summary of most significant groups within OASIS:}

- ebXML Committees--ebXML (Electronic Business using eXtensible Markup Language), sponsored by UN/CEFACT and OASIS, is a modular suite of specifications that enables ebusiness. Using ebXML, companies have a standard method to exchange business messages, conduct trading relationships, communicate data in common terms and define and register business processes. Significant technical committees are ebXML Business Process TC, ebXML Collaboration Protocol Profile and Agreement TC, ebXML Implementation, Interoperability and Conformance TC, ebXML Messaging Services TC, and ebXML Registry TC.

- UBL (Universal Business Language) -- intended to standardize the XML representation of basic business documents across many industries. The purpose of the UBL Technical Committee is to develop a standard library of XML business documents (purchase orders, invoices, etc.) by modifying an already existing library of XML schemas to incorporate the best features of other existing XML business libraries. Significant subcommittees are UBL Context Methodology SC, UBL Library Content SC, UBL Naming and Design Rules SC, and UBL Tools and Techniques SC.

- UDDI (Universal Description, Discovery and Integration) The UDDI specifications form the necessary technical foundation for publication and discovery of Web services implementations both within and between enterprises, providing Web services registry foundations. UDDI also allows operational registries to be maintained for different purposes in different contexts.

- Web Services Technical Committees - several Web Services TCs exist within OASIS defining and developing different ways to use Web Services. These TCs include Web Services Business Process Execution Language, Composite Application Framework, Web Services Distributed Management, Interactive Applications, Reliable Messaging, Remote Portlets, and Web Services Security.

- Public Key Infrastructure TC-- to address issues related to the successful deployment of digital certificates to meet business and security requirements as well as technical and integration/interoperability issues, and increase the awareness of digital certificates as an important component when managing access to network resources.

\footnotetext{
${ }^{20}$ BCF Workshop - Brisbane - EDOC 2003, Klaus Dieter Naujok, UN/CEFACT TMG Chair.
} 
- Domain specific TCs such as e-Government TC which demonstrates how OASIS can provide a forum for development and exchange concerning e-commerce needs of a specific sector.

\section{DISA}

\section{Summary of most significant groups within DISA:}

- $\quad$ ASC X12-- develops standards for cross-industry electronic exchange of business information, and active contribution to UN/CEFACT and other international e-business standards developing groups. Maintains the X12 EDI standards.

- $\quad$ X12C-Communications and Controls--Focuses on interchange and application control structures, control and management transaction sets, EDI architecture, data security, service specifications and other technical issues associated as they relate to the standards and their processes; develops X12's XML Reference Model and design rules; provides technical liaison on behalf of ASC X12 with national and international organizations involved in related activities.

- $\quad$ X12C Communications and Control-TG03 EDI-Architecture-Addressing semantics and architecture. In particular has developed the Context Inspired Component Architecture. CICA is supposed to be an architecture which is not vertically specific - but is cross-industry. An X12 reference model for XML design. It has many redundant and somewhat competing aspects with UBL and other ebXML related standards.

- $\quad$ ASC X12F Finance Subcommittee, Energy and Utilities Task Group - this is a very recently formed group (in liaison with UIG), initiating work on an X12 EDI transaction set to report and transmit metered service activity and information. This new transaction set will allow multiple meter accounts and sub-accounts at multiple billing rates to be reported in a single transaction. (NAESB contested the creation of Energy and Utilities Subcommittee in June 2003, it is possible that the scope of that group was then reduced and it was established as this task group within the Finance Subcommittee.)

- $\quad$ ASC X12J: Technical Assessment subcommittee - validates technical coherence of X12-related standards.

- COTG - Convergence and Outreach Task Group - a Task Group of the Steering Committee, handles education and outreach surrounding X12 standards work (marketing).

- DISA Registry Initiative - similar to other e-business registries, this is a prototype registry intended to include business process models, XML schemas and DTDs, and related business objects (such as industry-specific code lists) in a systematic way (it seems to be inactive in 2002 and 2003).

- DISA also maintains MOUs and joint initiatives which may be relevant to GridWise with groups such as CIDX and IFX - Interactive Financial Exchange Forum.

\section{A.1.6. Standards Organizations Details-IETF}

The Internet Engineering Task Force (IETF) is a long-standing, overarching, international Internet standards body. Its Internet Architecture Board may provide an effective gateway for GridWise architecture efforts to communicate with various specific efforts within the IETF. IETF has produced and maintains numerous key internet technology standards (such as TCP/IP), which GridWise communications rely on. Current IETF efforts are addressing many issues relevant to GridWise: EDI via internet, trade protocols, zero configuration and service discovery, secure and available credentials, network time-stamping, digital signatures, IPv6...and many others.

\section{Summary of most significant groups within IETF:}

- IAB-Internet Architecture Board. The IAB provides architectural oversight for IETF efforts and could be an effective interface between GridWise architecture and specific IETF efforts, as well as for integrating GridWise mindset into IETF endeavors.

- Application area: Electronic Data Interchange-Internet Integration (ediint). Develops and refines requirements and methods for packaging the EDI X12 and UN/EDIFACT transactions sets in a MIME 
envelope. Current efforts toward obtaining multi-vendor, inter-operable service revolve around security issues such as EDI transaction integrity, privacy and non-repudiation in various forms. Various technologies already exist for additional features and the primary requirement is to review and select a common set of components for use by the EDI community when it sends EDI over the Internet.

- Application area: Internet Open Trading Protocol (trade). The Internet Open Trading Protocol is an interoperable framework for Internet commerce. The working group is documenting interoperability experience with IOTP version 1 and developing the requirements and specifications for IOTP version 2 (using an independent Messaging Layer, standard XML Digital Signatures, and possibly dynamic definition of trading sequences, Offer Request Block, improved problem resolution, Ability to indicate and handle a payment protocol not tunneled through IOTP, support for wallet servers, repeated/ongoing payments, server to server messages, the ability to add both fields and attributes to trading blocks, collaboration with other protocols such as ECML).

- Internet area: Zero Configuration Networking (zeroconf). The goal of the Zero Configuration Networking (ZEROCONF) Working Group is to enable networking in the absence of configuration and administration. Zero configuration networking is required for environments where administration is impractical or impossible, such as in the home or small office, embedded systems 'plugged together' as in an automobile, or to allow impromptu networks as between the devices of strangers on a train.

- Internet area: Securing Neighbor Discovery (send). Neighbor Discovery is the basic protocol by which IPv6 nodes discover their default routers on the local link, and by which nodes on a local link resolve IPv6 addresses to MAC layer addresses, for delivery of packets on the local link, with the principle of enabling zero configuration, i.e., to allow hosts to start communicating with other hosts at the local link and in the Internet without any requirements for manual configuration. The objective of this working group is to define protocol support for securing IPv6 Neighbor Discovery without requiring manual keying.

- Security area: Securely Available Credentials (sacred). The working group is describing and identifying possible protocols in the areas of credential servers or "direct" transfer of credentials from one device to another (considering also security, availability, portability...), related architectural views and a standards-track document(s) describing the details of the adopted or developed protocol.

- Security area-- Secure Network Time Protocol (stime). For trust models to be truly portable across the Internet, transactions must be anchored so they are comparable. The ability to securely obtain time from authenticated sources is thus becoming a key factor in security and non-repudiation. The purpose of this working group is to define the message formats and protocols - specifically, modifications to the existing Network Time Protocol (NTP) - which are necessary to support the authenticated distribution of time for the Internet. The working group expects to enhance NTP by way of occasional "setup" interchanges between client and time server to establish a shared secret, followed by normal NTP interchanges secured via the shared secret.

- Security area--XML Digital Signatures (xmldsig). Digital signatures provide integrity, signature assurance and non-repudiatability over Web data. Such features are especially important for documents that represent commitments such as contracts, price lists, and manifests. In view of recent Web technology developments, the proposed work will address the digital signing of documents using XML syntax, critical for a variety of electronic commerce applications, including payment tools. The mission of this working group is to develop an XML compliant syntax used for representing the signature of Web resources and portions of protocol messages and procedures for computing and verifying such signatures. Such signatures may be able to provide data integrity, authentication, and/or nonrepudiatability. (JOINT IETF / W3C COORDINATION)

- Other IETF areas with aspects relevant to GridWise: Operations and Management, Transport, and Routing areas. (For example, IPv6). 


\title{
A.1.7. Standards Organizations Details-OMG \& W3C
}

Many of the specifications and methodologies developed by the World Wide Web Consortium (W3C) and the Object Management Group (OMG) have been embraced by international SDOs and widely adopted. Both of these groups have active, significant ties with various international SDOs.

It will be useful for GridWise architects to be familiar with the well established specifications of these two groups, and to monitor their progress in specific areas, to seek specific liaisons where appropriate as GridWise architecture is revealed.

Parts of the architectures, specifications, and languages of both the W3C and OMG may serve among building blocks to GridWise architectures.

\section{Summary of most significant groups within OMG:}

- Architecture Board - may act as gateway between GridWise architecture efforts and the OMG.

- Platform Technology Committee task forces and interest groups maintaining and extending existing OMG specifications, efforts which may be of interest to GridWise include:

\author{
- Middleware and Related Services PTF \\ - Real-time, Embedded, and Specialized Systems PTF \\ - Agents PSIG \\ ○ Legacy Transformation PSIG \\ - Ontology PSIG
}

- Domain Technology Committee task forces and interest groups in specific domains which may be addressing issues related to GridWise:
- Manufacturing Technology and Industrial Systems DTF
- Business Enterprise Integration DTF
- Telecommunications DTF
- eGovernment DSIG
- Super Distributed Objects DSIG
- Systems Engineering DSIG

\section{Summary of most significant specifications and resources within OMG:}

Unified Modeling Language (UML) - helps to specify, visualize, and document models of software systems, including their structure and design. UML is methodology-independent. UML defines twelve types of diagrams, divided into three categories: Four diagram types represent static application structure; five represent different aspects of dynamic behavior; and three represent ways you can organize and manage your application modules.

Model Driven Architecture (MDA) - The MDA is a new way of writing specifications and developing applications, based on a platform-independent model (PIM). A complete MDA specification consists of a definitive platform-independent base UML TM model, plus one or more platform-specific models (PSM) and interface definition sets, each describing how the base model is implemented on a different middleware 
platform. A complete MDA application consists of a definitive PIM, plus one or more PSMs and complete implementations, one on each platform that the application developer decides to support.

Common Object Request Broker Architecture (CORBA) - a longstanding middleware architecture which has been in use for many years. Upgrades and new features are still being added to CORBA.

Common Warehouse Model (CWM) - The Common Warehouse Metamodel (CWM $\left.{ }^{\mathrm{TM}}\right)$ is a specification that describes metadata interchange among data warehousing, business intelligence, knowledge management and portal technologies. The OMG Meta-Object Facility (MOFTM) bridges the gap between dissimilar meta-models by providing a common basis for meta-models. If two different meta-models are both MOF-conformant, then models based on them can reside in the same repository.

XML Metadata Interchange (XMI)-The 'new' OMG standard combining XML and UML. The XML Metadata Interchange Format (XMI) specifies an open information interchange model that is intended to give developers working with object technology the ability to exchange programming data over the Internet in a standardized way, thus bringing consistency and compatibility to applications created in collaborative environments.

Data Access Facility (DAF), Data Acquisition for Industrial Systems (DAIS), and Historical Data Access from Industrial Systems (HDAIS) - specifications for data access which are still used in certain industrial systems.

\section{Summary of most significant groups within W3C:}

Architecture domain supports XML, web services, internationalization, URI (Uniform Resource Identifiers), DOM (Document Object Models) development

Web Services--The World Wide Web is more and more used for application to application communication. The programmatic interfaces made available are referred to as Web services.

Web Services Architecture Working Group.

XML Protocol Working Group (SOAP, XML, XSL, XSLT...).

Web Services Description Working Group.

Web Services Choreography Working Group.

Semantic Web Services Interest Group.

Interaction domain projects are responsible for developing technologies that shape the web's user interface. These technologies include the (X)HTML, CSS, MathML, SMIL, SVG, VoiceXML, Multimodal Interaction and XForms.

Technology and Society Domain:

Semantic Web-- The Semantic Web is the representation of data on the World Wide Web. It is a collaborative effort led by $\mathrm{W} 3 \mathrm{C}$ with participation from a large number of researchers and industrial partners. It is based on the Resource Description Framework (RDF), which integrates a variety of applications using XML for syntax and URIs for naming.

OWL (Web Ontology) Working Group--The OWL Web Ontology Language is designed for use by applications that need to process the content of information instead of just presenting information to humans. 


\section{A.1.8. Standards Organizations Details-RosettaNet}

The RosettaNet standard forms a common e-Business language, aligning processes between trading partners on a global basis. It enables companies to carryout business-to-business (B2B) transactions in a seamless, secure, and real-time manner through system-to-system integration between customers and suppliers. RosettaNet PIPs (Partner Interface Processes) define business processes between trading partners and are specialized, system-to-system, XML-based dialogs. Each PIP specification includes a business document with the vocabulary, and a business process with a message dialog. The PIP approach can be used by GridWise architecture board to develop a similar standard for the GridWise community.

RosettaNet has great potential as a standard for automated e-Business. It has an effective focus on supply chain business processes. Many of RosettaNet's PIPs are already in production between trading partners in the IT, EC, and SM supply chains.

\section{Summary of most significant projects within RosettaNet:}

- Universal Messaging Service: RosettaNet developed the messaging services component, called the RosettaNet Implementation Framework Core Specifications, to supports its production implementations. RosettaNet Implementation Framework (RNIF) Core Specification is the packaging, routing, and transport of all PIP ${ }^{\circledR}$ messages and business signals, it define the manner in which execution of RosettaNet's PIP specifications may occur. The RNIF 2.0 includes a transfer protocol-independent container that packages together the business payload along with the header components and other elements that all must be exchanged between two participants in a RosettaNet e-business standard interaction.

- $\quad$ RosettaNet Partner Interface Processes ${ }^{\circledR}\left(\operatorname{PIPs}{ }^{\circledR}\right)$ define business processes between trading partners. PIPs are specialized system-to-system XML-based dialogs. Each PIP specification includes a business document with the vocabulary, and a business process with the choreography of the message dialog. PIPs apply to the following core processes: Administration; Partner, Product and Service Review; Product Introduction; Order Management; Inventory Management; Marketing Information Management; Service and Support; and Manufacturing.

- $\quad$ RosettaNet Business Dictionary (RNBD) defines the Business Properties, Business Data Entities, and Fundamental Business Data Entities in PIP Message Guidelines.

- $\quad$ Global Company Identifier Program--addresses issues surrounding the use of Data Universal Numbering System (D-U-N-S®) for Global Company Identifier in RosettaNet PIPs ${ }^{\circledR}$. RosettaNet specifies the Global Trade Item Number (GTIN) for Global Product Identifier in its PIPs. The GTIN is a worldwide multi-industry standard for trade-item identification. GTINs are 14-digit numbers that uniquely and globally identify products and services. UN/SPSC for Global Class Identifier, a code standard for classifying products and services.

The RosettaNet standard forms a common e-Business language, aligning processes between trading partners on a global basis. It enables companies to carryout business-to-business (B2B) transactions in a seamless, secure, and real-time manner through system-to-system integration between customers and suppliers. RosettaNet PIPs (Partner Interface Processes) define business processes between trading partners and are specialized, system-to-system, XML-based dialogs. Each PIP specification includes a business document with the vocabulary, and a business process with a message dialog. The PIP approach can be used by GridWise architecture board to develop a similar standard for the GridWise community. 


\section{A.1.9. Standards Organizations Details-EIA \& CEA \& TIA}

Although the Electronic Industries Alliance (EIA) does not have its own standards developing committees, all work is done in the various associations - Consumer Electronics Association (CEA),

Telecommunications Industry Association (TIA) or others, as the official United States Technical Advisory Group (US TAG) in certain areas, they are highly responsible for standards development within the ISO/IEC JTC 1/SC25, the key international SDO committee establishing international home and building automation standards, covering:

- $\quad$ HomeGate: A residential gateway to link home control networks with external service provider networks (WAN/LAN translating).

- Application Interoperability methods and models: interoperability to allow home system applications created by different manufacturers to communication with each other (lighting, security, energy management...) to allow integrated home systems.

- Universal Interface: An interface module to be incorporated into an appliance for communicating over a variety of home automation networks.

- $\quad$ Broadband Home Network: based on Versatile Home Network (EIA-851).

- Security and privacy: to add encryption and authentication to communications both within the house and via the Internet.

- $\quad$ Functional safety: product interaction via a home control network requires a harmonization of safety requirements.

- Structured cabling: A Joint Protect Team of SC25/WG1 and SC25/WG3 (Building Wiring) is writing a standard for integrated residential wiring, covering topology, applications, descriptive and prescriptive aspects.

The Consumer Electronics Association (CEA) has a Home Networking and IT Division addressing general issues, but standards development of significance for GridWise is done within the subcommittees of the R7 Home Network Committee, and their working groups:

- $\quad$ R7 Home Network Committee: R7 provides coordination for, and encourages cooperation among, all CEA home network standardization efforts as well as providing a forum for non-CEA Home Network standards formulating bodies interested in working with CEA, to ensure current and future Home Networks can coexist within a home and share information through the use of industry standard interfaces primarily related to the interconnection and coexistence of home networks.

- R7.1 Home Controls Subcommittee 1 (HSC1 Subcommittee): R7.1 standardizes a home control networking protocol based on the LonTalk ${ }^{\circledR}$ protocol (ANSI/EIA/CEA-709.1 Control Network Protocol). LonTalk supports a variety of physical media and is based on Echelon's proprietary neuron-chip implementations and supports relatively high data transfer rates (encompasses OSI layers 1,2,3,4,5,6, and7).

- R7.2 CEBus (Consumer Electronic BUS): The Consumer Electronic Bus (CEBus) standard is a control communication protocol, presently used for home automation applications such as home lighting systems, security systems, appliance control and monitoring (including entertainment equipment such as sound systems), and also in utility and commercial sector applications (EIA-600 Home Plug and Play). CEBus supports a variety of physical media, is not based on proprietary implementations, and has relatively limited data transfer rates (encompasses OSI layers 1,2,3,7). (EIA/CEA 600 CEBus Standard, and EIA/CEA 721 Generic Common Application Language.)

○ R7.4 Versatile Home Network Subcommittee: R7.4 develops and maintains standards for 
interoperability between applications on a common in-home distribution network for consumer electronics, using Internet protocols and IEEE 1394 technology (also known as Firewire or i.Link). These standards address interconnection of various device clusters and residential gateways (VHN EIA-851).

The Telecommunications Industry Alliance (TIA) has several technical committees for specific telecommunications and cabling issues, and TIA is active on the US TAGs for some IEC Technical Committees. At this stage, no committees of particular significance for GridWise have been identified, although structured cabling may be relevant and TR 41 and TR-42 User Premises Telecommunications Infrastructure (TIA 862) do include information aspects within their scope.

\section{A.1.10. Standards Organizations Details-OSGi}

The Open Services Gateway initiative (OSGi) provides a series of specifications for home gateway integration-permitting a single platform handling multiple in-home services interfaced to the outside world through various possible communications technologies. These specifications can be compared and contrasted to the HomeGate standardization work currently underway in the IEC/ISO JTC1/SC25 (with the participation of the EIA \& CEA) - they might be seen as one particular rendition and subset of this type of standard.

The past decade has seen growth in many different standards and technologies surrounding home networking, including both proprietary and consortia developed systems. OSGi was a visible consortia addressing the subject of home service gateways, with a wide industry membership base, and a certain number of products in industry adhering to the OSGi specifications. However, the subject of home gateways seems to be maturing and entering the international standards organizations, so the strength and leveraging potential of OSGi from the viewpoint of GridWise needs may be diminishing.

\section{Summary of most significant groups within OSGi:}

- Architecture Expert Group--The Architecture Expert Group (AEG) is a little different from the other highly specific EGs. It acts as the co-ordination function and it is through the AEG that new work items are brought into the community. If someone wants to develop a new expert group to address a specific area of application of OSGi, for example, this is done via the AEG. In addition the AEG acts as the home for the Technical Steering Committee, made up of the chairs of all of the other EGs, which co-ordinates the actions between them.

- Core Platform Expert Group--The Core Platform Expert Group (CPEG) defines the core platform specifications and APIs that the OSGi Alliance requires. The CPEG is focused on components that form the execution environment for OSGi services and provides the fundamental services for all OSGi environments.

\section{A.1.11. Standards Organizations Details-(AHAM)}

The recent work done by the Smart Appliance Task Force has the advantage of being completely nonproprietary, product independent, and developed with the participation of key appliance manufacturers (Whirlpool, GE, Maytag...).

Some of the primary participants in this standards development with the Association of Home Appliance Manufacturers (AHAM) are also active in other building automation standards efforts: BACnet, LonTalk, OSGi, UPnP.

This work presents a key generic model for fitting appliances into building automation systems. 


\section{Summary of most significant groups within AHAM:}

Appliance Research Consortium (ARC): partnership of appliance manufacturers, DOE and EPA; board authorizes all research projects and establishes Technical Advisory Committees to oversee specific projects.

- Smart Appliance Task Force: Task Force responsible for developing the key standard Connected Home Appliances-Object Modeling (ANSI/AHAM CHA-1-2003)—objectoriented generic models of key appliances to facilitate linking in home network systems.

- Appliance Technology Roadmap: in theory a project between AHAM (ARC) and DOE-no written trace of results from this project have been identified.

\section{A.1.12. Standards Organizations Details-ASHRAE}

American Society of Heating, Refrigerating and Air-Conditioning Engineers (ASHRAE) is a professional society that plays a significant role in the development of voluntary standards for HVAC systems and energy efficiency. There are several technical committees, standards project committees and research projects of interest to GridWise. The BACnet standard, XML definitions for HVAC equipment and some of the research projects could provide useful information for the GridWise architecture. It is also possible to collaborate with ASHRAE by participating in technical committees and other activities to get industry input for the GridWise architecture development.

Several people within PNNL are active members of ASHRAE and have participated in the past in ASHRAE efforts related to the GridWise scope. Given the extent of readily available human resources and given the international penetration of BACnet (ISO TC205 adoption), ASHRAE could have strong leveraging potential for GridWise (at relatively low cost).

ASHRAE's organization differentiates between Guideline Project Committees (GPC), Standards Project Committees (SPC), and Technical Committees (TC). There are committees of each type which are relevant to GridWise.

\section{Summary of most significant groups within ASHRAE:}

- GPC-20: XML Definitions for HVAC\&R--GPC-20 is developing a common data exchange format for the description of commodity data and HVAC\&R information using the standard XML formatting language. Data types would include catalog definitions in areas such as chillers, air-handling units, fans, pumps, fittings, controls, as well as analytical or operations, building performance data.

- SPC 135: BACnet - A Data Communication Protocol for Building Automation and Control Networks-The BACnet standard defines data communication services and protocols for computer equipment used for monitoring and control of HVAC\&R and other building systems. It also defines an abstract, objectoriented representation of information communicated between such equipment.

- SPC 111: Practices for Measurement, Testing, Adjusting and Balancing of Building Heating, Ventilation, Air-Conditioning and Refrigeration Systems This standard provides methods for evaluating building heating, ventilation, air-conditioning and refrigeration systems.

- Technical Committees - Section 1.0 - Fundamentals and General

- TC 1.4: Control Theory and Application--concerned with determination of control theory, systems, and components for heating, ventilating, air-conditioning and refrigeration uses

- TC 1.5: Computer Applications--concerned with determination of computer applications to the design and optimization of refrigeration, heating, and air-conditioning systems, equipment and components; and with programs for all such uses. 
- Technical Committees - $\underline{\text { Section } 7.0 \text { - Building Performance }}$

- TC 7.4: Building Operation Dynamics--concerned with the dynamic characteristics and interactions of comfort conditions, the active components of HVAC systems, control systems, and operation strategies of a building.

- TC 7.5: Smart Building Systems--concerned with the performance and interactions of smart building systems, the impact of smart systems on the total building performance, methods for achieving more intelligent control and operation of building processes, interactions of smart buildings with utilities.

- TC 7.6: Systems Energy Utilization--concerned with procedures for accounting for all types of energy usage in structures and quantifying these energy usages as a function of the character and utilization of the structure.

\section{A.1.13. Standards Organizations Details-FIATECH}

The Fully Integrated and Automated Technologies consortium (FIATECH) focuses on deployment of emerging and new technologies to the capital projects industry. One of the FIATECH objectives is to improve the data and information management by developing or adopting interoperable data models. AEX is one of the initiatives that is relevant to GridWise. AEX data model encompasses building HVAC and mechanical equipment data for business processes. This data model integrated with operation and maintenance information could be used by the GridWise architecture.

\section{Summary of most significant projects within FIATECH:}

- $\quad$ AEX - Automated Equipment Information Exchange-- to enable both internal and external automated information exchanges among the multiple software systems and collaborating companies associated with design, procurement, fabrication, delivery, installation, operation and maintenance of engineered equipment items (Collaborating with ASHRAE GPC-20 on developing a guideline document 'XML Definitions for HVAC').

- Data Standards and Open Systems Resource-- information service to FIATECH members to help them with a list of all the facility related data standards and standards organizations.

- Capital Projects Technology Roadmap-- to promote a highly automated project and facility management environment integrated across all phases of the facility life cycle. Planning and scheduling systems, financial systems, design systems, job site management and control systems, and other functions will interact with a master model to acquire and provide the information they need to perform their functions. Links from the master model to external information sources will enable scheduling and design applications, procurement systems, etc. to quickly access the outside information they need to accomplish their tasks.

\section{A.1.14. Standards Organizations Details-ISO}

The Internationals Standards Organization (ISO) carries considerable weight and respectability, like the IEC. Although the ISO does not focus on the electro-technical domain like the IEC, it nevertheless has many higher lever initiatives related to GridWise, and is joined with the IEC for all JTC1 efforts (home electronic systems, OSI, Open Distributed Processing, OPEN EDI....). ${ }^{21}$ The ISO also works jointly with the UN/CEFACT (EDIFACT) on some efforts concerning electronic commerce and EDI.

\footnotetext{
${ }^{21}$ IEC/ISO JTC1 efforts are listed in the IEC section of this report, A.1.1.
} 
In direct relation to communication protocols in the power sector, ISO TC 184 on industrial automation systems is responsible, in particular, for the MMS-Manufacturing Messaging Specification (ISO 9506), which was the historical basis for ICCP (TASE.2) and initial UCA protocols. Subcommittee 5 of TC184 covers architecture, communications and integration framework for systems involving real-time control, complex systems, and integration of numerous business actors. The current projects within TC184 cover numerous areas related to GridWise, including: system modeling, device control, interoperability and integration, distributed control systems, and electronic commerce (EDI coordination) — often at a generic, not domain-specific level.

Beyond industrial automation systems, ISO subgroups also cover other sectors within the GridWise scope, including data exchange standards for the financial trading sectors and building automation systems. ISO also encompasses many subjects that are indirectly related to GridWise, like Geographic Information Systems, or areas may provide examples and parallels for GridWise such as Intelligent Transport Systems.

\section{Summary of most significant sub-groups within ISO:}

- ISO/IEC JTC1: Please see IEC details for this information.

- TC 68 Banking, securities and other financial services

- ISO/TC 68/SC 2 - Security management and general banking operations (Framework for IT security for financial institutions, Public key infrastructure management for financial services)

- ISO/TC 68/SC 4 - Securities and related financial instruments (ISO 15022-- SWIFT, EDIFACT syntax, integrates XML, also)

- TC 184 Industrial Automation Systems, SC 5--Architecture, communications and integration frameworks (ISO9506 MMS)

- TC 184/SC 5/WG 1-Enterprise modeling and architecture (ISO 14258, 15704, 19439, \& 19440)

$\circ$ TC 184/SC 5/WG 2-Communications and interconnections (ISO 13283)

○ TC 184/SC 5/WG 5-Open systems application frameworks (ISO 15745)

- TC 184/SC 5/WG 6-Application service interface

- TC 184/SC 5/JWG 15-Joint ISO/TC 184/SC 5 - IEC/SC 65A WG : Enterprise-control system integration (ISO 62264)

- TC 205 Building Environment Design

- TC 205/WG 3 Building control systems design (Building Automation Protocols) internationalization of BACnet efforts (ISO 16484)..

\section{Other efforts which may be of interest to GridWise Architecture:}

- TC 184 Industrial Automation Systems, SC 4-Industrial data. (ISO 10303 series STEP)

- TC 204 Intelligent transport systems. Parallels and examples of information, communication and control systems in the field of urban and rural surface transportation, including wide area communications, electronic identification and payment systems, distributed monitoring and control.

- TC 154 Processes, data elements and documents in commerce, industry and administration. Meta-standards for data specification and process identification, in conjunction with 


\section{UN/CEFACT.}

- TC 211 Geographic information/Geomatics. Conceptual schemas, models, meta-data for standardized interfacing and integration of GIS.

- Some TCs which may have an indirect bearing at some point in the future on specific distributed generation or load control (TC 70 Internal combustion engines, TC 86 Refrigeration and air-conditioning, TC 192 Gas Turbines...)

\section{A.1.15. Standards Organizations Details-OPC \& ISA}

The OLE for Process Control Foundation (OPC) inner workings and organizational structure are not visible to non-members on the OPC Foundation website, but it would appear that working groups are defined as needed for any given existing or emerging specification. OPC stands for OLE Process Control, that is Object linking and embedding Process Control.

\section{Summary of most significant specifications within OPC:}

- OPC Data Access (OPC DA): The original OPC specifications, used to move real-time data from PLCs (Programmable Logic Controller), DCSs (Distributed Control Systems), and other control devices to HMIs and other display clients or SCADA systems.

- OPC Alarms \& Events: Provides alarm and event notifications on demand (in contrast to the continuous data flow of Data Access).

- OPC Batch: Provides interfaces for the exchange of equipment capabilities (corresponding to the S88.01 Physical Model (pre-S95, ISO62264)) and current operating conditions. Working toward plug and play capacities of batch processing components.

- OPC Data eXchange (OPC DX): Provides an industry standard for exchanging data between OPC Data Access servers, to define server-to-server and device-to-device communication across Ethernet Fieldbus networks, adding remote configuration, diagnostic and monitoring/management services (horizontal movement of data between control platforms).

- OPC Historical Data Access: Provides access to data already stored, from a simple serial data logging system to a complex SCADA system, historical archives can be retrieved in a uniform manner.

- OPC Security: Specifies how to control client access to OPC servers in order to protect this sensitive information and to guard against unauthorized modification of process parameters.

\section{More recent and emerging OPC Specifications include:}

- OPC XML-DA: Rules and formats for exposing plant floor data as in original OPC Data Access Specifications by using XML, including definitions for supporting Web Services.

- OPC Complex Data (in progress): A companion specification to Data Access and XML-DA that allows servers to expose and describe more complicated data types such as binary structures and XML documents.

- OPC Commands (in progress): Developing a new set of interfaces that allow OPC clients and servers to identify, send and monitor control commands which execute on a device.

Summary of most significant groups within the Instrumentation, Systems \& Automation Society (ISA):

- ISA-SP95, Enterprise/Control Integration Committee--Define in detail an abstract model of the enterprise, including manufacturing control functions and business 
functions, and its information exchange, common terminology for the description and understanding of enterprise (ANSI/ISA-95 series, basis for ISO 62264 on enterprise control system integration).

- ISA-SPTAG65, Industrial-Process Measurement and Control--To prepare international standards for systems and elements used for industrial-process measurement and control concerning continuous and batch processes as Technical Advisory Group (USTAG) for IEC TC65.

- ISA-SPTAG65A, System Aspects--To prepare standards regarding the generic aspects of systems used in industrial-process measurement and control: operational conditions (including EMC), methodology for the assessment of systems, functional safety, as the Technical Advisory Group (USTAG) for IEC SC65A.

- ISA-SP99, Manufacturing and Control Systems Security

- ISA-SP72, Industrial Computer Interfaces and Data Transmission Techniques -Interfaces and data transmission techniques between computers and subsystems used in industrial process control (Manufacturing Message Specification-MMS).

- ISA-SP88, Batch Control--Standard batch control architecture that defines both the physical model and the functional model. The physical model is the hierarchical structure that relates control equipment and data communications needed for the physical areas involved in batch control. The functional model shows the relationships between the five types of control recipe management, scheduling, sequential control, regulatory control, and safety interlock systems (lower level control below the SP95 work).

Also ISA has various TCs for operational control of power plants, turbines, control centers, ISA-SP50, Signal Compatibility of Electrical Instruments (ANSI/ISA 50.02 Fieldbus standards).

The OPC Foundation (OLE for Process Control Foundation) and ISA Society (Instrumentation, Systems and Automation Society) recently agreed to establish a joint working group (along with MIMOSA) to develop open enterprise integration standards for operations and maintenance applications. Open integration requirements for applications such as Manufacturing Execution Systems, Operations Data Historians, Human Machine Interfaces, Enterprise Resource Planning, Enterprise Asset Management and Condition Based Maintenance will be addressed by the effort.

ISA SP95 defines information and process models for information flow, essentially defining the 'what', while OPC defines the 'how' to move the data and information (although OPC is somewhat centered around OLE/Microsoft-based software environments) for industrial automation. OPC has a solid presence in industry automation, and its recent specifications to encompass XML and Web Services compatibility demonstrate that it is still progressing. ISA technical committees involved with industrial systems and controls developed some significant standards in the past, though it is not clear from their website which technical committees are truly active today. Nevertheless, the recent creation of the joint working group between OPC, ISA and MIMOSA would seem to indicate that ISA SP95 is still working actively.

\section{A.1.16. Standards Organizations Details-ITI-INCITS}

The Information Technology Industry Council's InterNational Committee for Information Technology Standards (ITI-INCITS) and its committees presented below may be significant for GridWise primarily because of their roles as US TAGs (technical advisory groups) to important international standards efforts, particularly within the ISO/IEC JTC1, and for the high-level nature of the issues they tend to address. These committees tend to address the more architectural, guiding aspects of key IT areas (databases, GIS, metadata, open distributed processing, and security techniques).

- H2 - Technical Committee on Database. Ties between legacy systems and more recent database 
technologies, US TAG to ISO/IEC JTC1 SC32/WG3, and WG4.

- L1 - Geographic Information Systems (GIS). Projects: Numerous GIS related projects underway, US TAG to ISO/TC 211.

- L8 - Metadata (also applies to Home Electronic Systems). Considerable work in Electronic Commerce and EDI, standardizing and mapping to permit separate IT worlds to interrelate, Metadata and Metadata Registries. US TAG to several committees.

- T3 - Open Distributed Processing (ODP). Develop a Reference Model of Open Distributed Processing (RM-ODP) to coordinate the development of distributed processing standards and related standards for components of ODP, which includes ASN.1, Formal Definition Techniques, OSI System Management and OSI Directory Services. US TAG for ISO/IEC JTC1 SC 6.

- T4 - Security Techniques. Work done in liaison with IETF on network security, Security Management and General Banking Operations. US TAG to several committees.

\section{A.1.17. Standards Organizations Details-ATIS}

The Alliance for Telecommunications Industry Solutions (ATIS) does not work on many specific standards directly related to GridWise Architecture, but it holds significance for GridWise because of the parallels between the issues they address as public service providers, for standards surrounding telecommunications infrastructure, and for their role as US TAG in the telecommunications domains.

ATIS-T1 is a key accredited US SDO for telecommunications. They have standards efforts covering specific technologies related to GridWise: telecommunication channels for command, control, and communications.

Representing a sector which shares some characteristics of the Utility industry (wide-area public service), the experience and progress of ATIS-T1 may provide useful insight for GridWise architecture needs. ATIST1 efforts cover issues including public service, local, regional, national issues, QoS issues, interconnections, safety, providers/users. They are also in the process of applying overarching architectural concepts--ODP, network layer concepts...--to the existing world of older telecommunications standards.

Finally, ATIS has liaisons with other standards making groups which are of interest to GridWise (ITU-T and IEC/JTC-1).

\section{Summary of most significant groups within ATIS:}

- Committee T1 - Telecommunications (ASC T1) focuses on functions and characteristics to interconnect and interoperate communications networks and operation support systems at interfaces with end-users and carriers and with information, Internet, and enhanced service providers, works with the ITU-T and ISO/IEC JTC1.

○ T1E1-- Interfaces, Power \& Protection for Networks T1E1.5-- Power Systems Working Group : interconnection of power systems, the components of power systems, the characteristics of power system interfaces used with telecommunications load equipment.

- T1M1.5-OAM\&P Architecture, Interface and Protocols: addresses Operations, Administration, Maintenance and Provisioning (OAM\&P) related to the management of telecommunications networks, specifically the Telecommunications Management Network (TMN) architecture, applications functions \& requirements, information modeling related to the five OSI Management Functional Areas (MFAs) and planning related to the migration of TMN to Open Distributed Processing (ODP) concepts per ISO/IEC 10746 parts 1-4 (X.901-X.904), building on the application of ODP to management of transmission network by ITU-T SG 4 in G.851-xx documents. 
- Telecommunications Industry Forum (TCIF): promotes electronic commerce, electronic data interchange, improvements in bar coding of telecommunications products for inventory control, and electronic bonding.

- Electronic Ordering: analyzes business requirements for the purpose of assisting with the creation and revision of standard interfaces for access and local ordering services, and identifies, analyzes, resolves, and documents technical and operational issues for the implementation of Electronic Ordering interface standards.

- EDI Service Order Committee (ESOC): an industry group consisting of telecommunications service providers and their customers working together to develop Business Models and Industry Guidelines for the electronic ordering of telecommunications goods and services via EDI.

- EDI Telecommunications Billing (ETB): an industry group comprised of representatives from telecommunications providers and customers. ETB develops telecommunications Business Models and Implementation Guidelines standardized for industry use of: 811 Consolidated Service Invoice/Statement, 820 - Payment Order/Remittance Advice, 864 Text Message.

\section{A.1.18. Standards Organizations Details-ANSI}

None - the American National Standards Institute (ANSI) serves in the accrediting of standards organizations, as a publishing clearinghouse, and administrative aspects surrounding US standards participation; but ANSI does not develop standards. 
Appendix B

General Background on Standards-Related Organizations 


\section{B. General Background on Standards-Related Organizations}

There is no clear-cut, accepted ontology for classifying the various types and roles of standards-related organizations, and no simple, commonly agreed upon ranking as to the significance or importance of various organizations. The realm of standards-developing organizations has evolved greatly in recent years, with the proliferation and increasing impact of more informal types of organizations like industry consortia. This evolution has been driven by many factors, in particular the expansion of information technology sectors (driving greater needs for standards), and the Internet and its associated facilitation of communication both toward dispersed communities of stakeholders and within dispersed working groups. Twenty years ago, standards making occurred in a few, accredited international standards organizations and in government-sponsored (or accredited) national standards bodies. Today, those bodies continue to function, but are now obliged to cooperate, complement and compete with quite an array of other standards-related organizations. ${ }^{22}$

\section{Standards-Developing Organizations (SDOs) evolve with Industry}

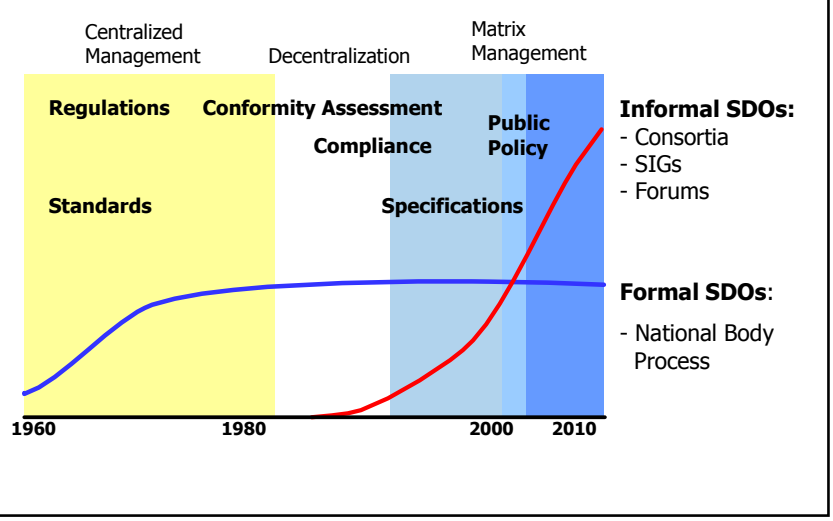

\section{B.1. General Categories of Standards Organizations}

Standards researchers identify many different types of standards-related organizations (the delineations are not clear cut and there are many overlaps among these categories):

- 'Official' SDOs (Standards-Developing Organizations):

- International SDO's (the ISO, IEC and ITU $^{23}$ ), which generally function on the principle of one country/one vote.

- National Standard Bodies, generally one principal NSB per country (in the US it is ANSI) - federates, coordinates, represents and administers, but does not usually develop standards.

- Regional SDOs (government accredited, spanning a group of countries, for example, Europe, North America, Asia, the Americas, etc.).

\footnotetext{
${ }^{22}$ Illustration adapted from 'Presenting ECMA International', ECMA-European Computer Manufacturers Association International. Available online URL: www.ecma-international.org. 2003.

23 Some specific definitions would say that the ITU is not an International SDO because it does not function on the basis of one country/one vote - although in most other ways it can be seen as an official international SDO.
} 
- Nationally Accredited SDOs ( 270 business and industry, standards developers, trade associations, labor unions, professional societies, consumers, academia, and government agencies accredited by ANSI).

- Accredited Committees and Sub-groups of International and National SDOs.

- 'Recognized' SDOs (also 'official', in slightly different manners):

- National or International SDO Affiliated or Designated Organizations (for example, designated to maintain or develop specific standards or codes, including for example, the IEEE or UCC).

- Recognized Organizations (consortia, trade groups, professional societies whose impact and contributions are so great that National and/or International SDOs recognize them and work cooperatively with them) these consortia are usually international, with formal liaisons to international SDOs.

- Standards Developing Groups (many of these organizations are also quite recognized now, with structured relationships with other, more formal SDOs):

- Industry specific or cross-industry consortia, trade groups or professional societies.

- Special interest groups, Initiatives.

- Forums.

- Private sector companies.

- Standards Influencing Groups:

- Industry trade groups (with policy influencing objectives, like the ITI).

- Special interest groups for general promoting or promoting implementation of specific standards and technologies (like user groups)

- Private sector companies.

Many standards-related organizations have internal Technical Advisory Groups (TAGs). It is important to note that the term 'US Technical Advisory Group' specifically relates to subcommittees (usually hosted by ANSI Accredited SDOs), which represent the US (via ANSI coordination) in specific ISO and IEC technical areas. Participation in US TAGs is the officially recognized means for US input to enter the international standardization arena.

\section{B.2. General Characteristics of Standards-Related Organizations}

The standards-related organizations that fall into all of these different categories often have both similarities and differences, which do not necessarily correspond to any given category. For example, some formal SDOs have adopted principles of open standards, as have many recognized consortia, whereas some still maintain some very closed processes.

Table B.1 below summarizes some of the ranges of issues that characterize standards-related organizations. 


\begin{tabular}{|c|c|c|}
\hline & Issues & Range \\
\hline \multirow{10}{*}{ 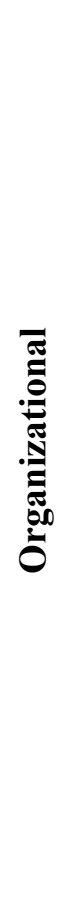 } & 'Official-ness' & Restricted $\rightarrow$ Non-accredited $\rightarrow$ Accredited \\
\hline & Participation & $\begin{array}{l}\text { Closed } \rightarrow \text { Open } \\
\text { Individual } \rightarrow \text { Company } \rightarrow \text { Country }\end{array}$ \\
\hline & Consensus & Simple Majority $\rightarrow$ Unanimity \\
\hline & Market Place & Local $\rightarrow$ National $\rightarrow$ Regional $\rightarrow$ International \\
\hline & Maintenance & Onetime $\rightarrow$ Ongoing \\
\hline & Time/Speed & Slower $\rightarrow$ Faster \\
\hline & Process & Informal development process $\rightarrow$ Formal process \\
\hline & Funding Source & Government $\rightarrow$ Dues $\rightarrow$ Publications \\
\hline & Cost & $\$ \rightarrow \$ \$ \$$ \\
\hline & Visibility & Unknown $\rightarrow$ Brand name recognition \\
\hline \multirow{6}{*}{ 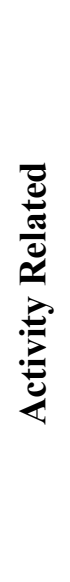 } & Phase of Standards Work & $\begin{array}{l}\text { Conceptual } \rightarrow \text { Anticipatory } \rightarrow \text { Participatory } \rightarrow \\
\text { Responsive }\end{array}$ \\
\hline & Applicability & Convention $\rightarrow$ De facto $\rightarrow$ Published \\
\hline & Specificity & Specific \& narrow $\rightarrow$ Abstract $\&$ broad \\
\hline & Horizontal Breadth & Sub-sector $\rightarrow$ Cross-industry \\
\hline & Vertical Depth & $\begin{array}{l}\text { Single step, process, or product } \rightarrow \text { Supply chain } \rightarrow \text { Life- } \\
\text { cycle }\end{array}$ \\
\hline & Technical Focus & Single layer $\rightarrow$ Multiple technical layers \\
\hline
\end{tabular}

The background explanations in this document do not attempt to coin new definitions for standards and SDOs where even the research community has no coherent, globally adopted terminology. However, this discussion highlights a few points:

- It is useful to recognize that a variety of definitions exist for standards and SDOs.

- Multiple, qualitative factors must be taken into consideration when analyzing and comparing standards and SDOs.

- Each factor may or may not be significant - an intangible accumulation of factors will help determine the importance of various standards to GridWise architectural efforts. 


\section{B.3. General Background on Standards}

A standard can be defined as a set of technical definitions and guidelines, "how to" instructions for designers, manufacturers and users. Standards promote safety, reliability, productivity and efficiency in almost every industry that relies on engineering components or equipment. Standards can run from a few paragraphs to hundreds of pages, and are written by experts with knowledge and expertise in a particular field who sit on many committees. Standards are a vehicle of communication for producers and users. They serve as a common language, defining quality and establishing safety criteria. Costs are lower if procedures are standardized; training is also simplified.

Standards are considered voluntary because they serve as guidelines, but do not of themselves have the force of law. Standards organizations cannot force any manufacturer, inspector, or installer to follow standards. Standards become mandatory when they have been incorporated into a business contract or incorporated into regulations. A code is a standard that has been adopted by one or more governmental bodies and has the force of law.

At different phases in the birth and life of a service or product, standards may be developed for different purposes. From a concept or idea, anticipatory standards may be drawn up. As markets emerge, participatory standards emerge corresponding to products or services. As markets collide with existing markets and regulatory needs are felt, responsive standards are established. All of these phases of standards are influenced by environmental factors, including technology, regulatory environments, markets, and architectural guidelines (if they exist). ${ }^{24}$

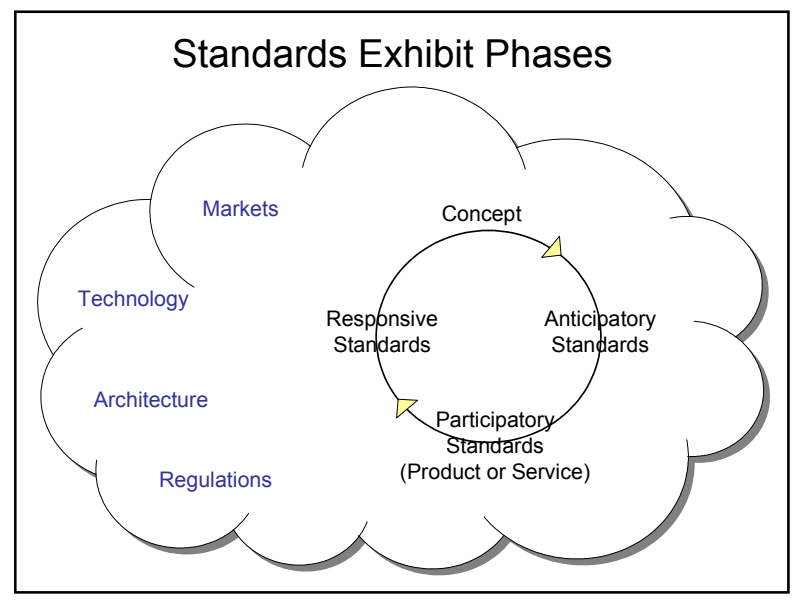

National standards bodies, like ANSI, tend to define standards as only those emitted by 'accredited' standards bodies. They term de facto standards as 'conventions'. In other sectors, broadly accepted guidelines are often termed as 'standards'; for example, we now often read about 'Web Standards' of the W3C (whereas the W3C does not 'officially' publish standards). Many professionals would say that it is not whether a standard is emitted by an 'accredited' body that counts, but the extent of its actual acceptance and use. Regulatory and judicial bodies (defining codes and interpreting law) tend to give more credence to standards developed through 'accredited' bodies, but also recognize and draw from standards work of 'non-accredited' groups.

\footnotetext{
${ }^{24}$ Illustration adapted from Krechmer 2002.
} 


\section{B.4. A General Picture of the Standards-Related World}

The diagram below depicts the general layout of the standards-related world, differentiating between international, regional and national bodies, accredited and non-accredited, and standards development organizations (SDOs) as opposed to influence groups less involved in standards development.

The groupings and delineations are illustrative, but readers should remember that these terms have different connotations for different professionals - depending on an individual's relationship to the standards-making process and depending on industry sectors. 'Officialness' and accreditation does not necessarily reflect a standard's impact.

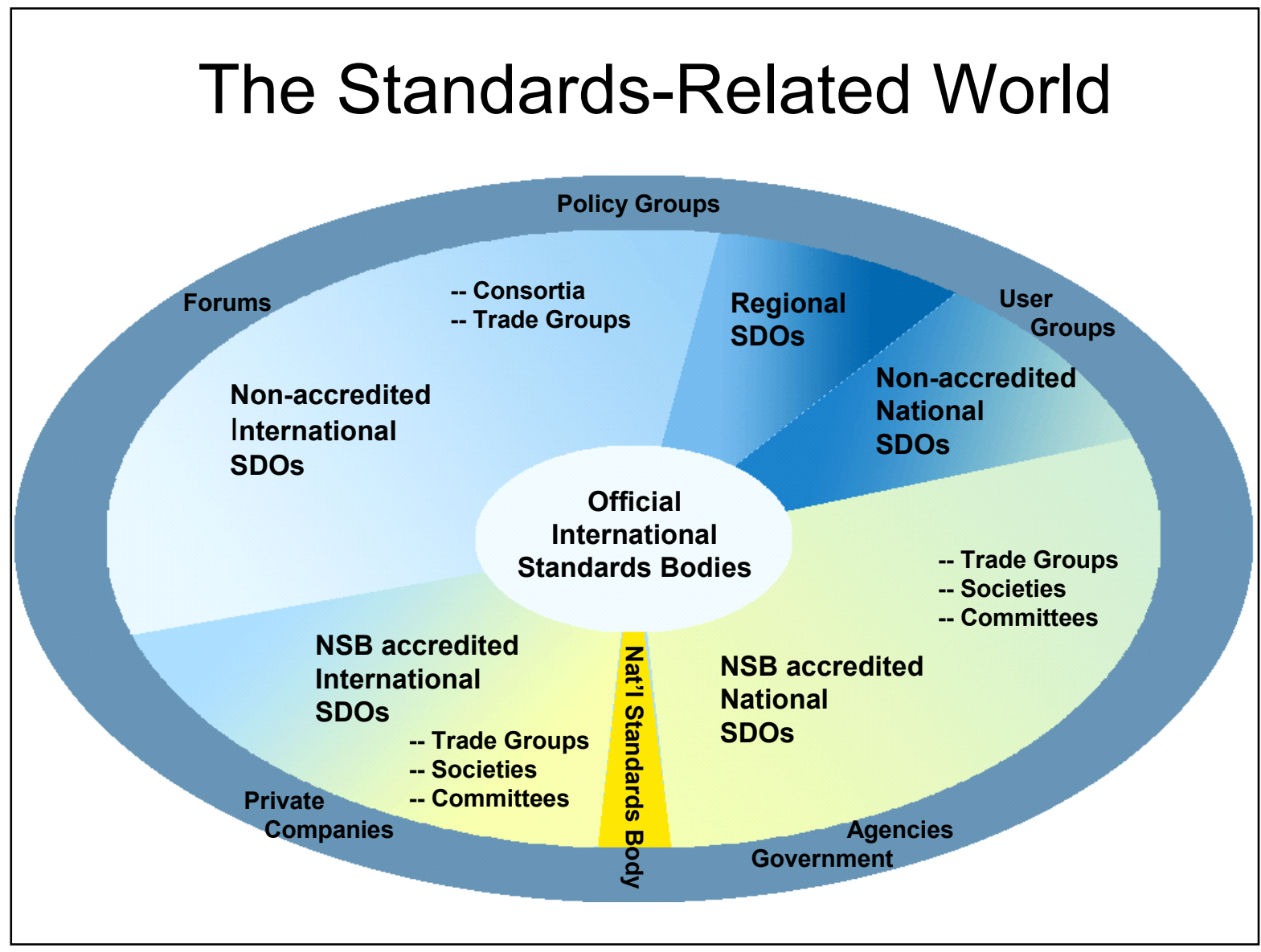




\section{B.5. A General Picture of GridWise-Related SDOs}

To summarize the list of standards-related organizations included in Appendix A, the diagram below presents these specific organizations of the standards-related world, mapping the acronyms of SDOs related to GridWise into the schema of the Standards-Related World.

ANSI is included as the United State's Official National Standards Body. Although it does not develop standards, it federates accredited standards developing organizations and represents the US in official international SDOs.

Some readers might argue that the only truly international SDOs are the ISO and the IEC, because the ITU accepts members that are not countries and the UN/CEFACT is not at its origin a standards developing organization.

The international SDOs (both accredited and non-accredited) are the most diverse group — with a variety of sizes, formats, funding mechanisms, and standards processes.

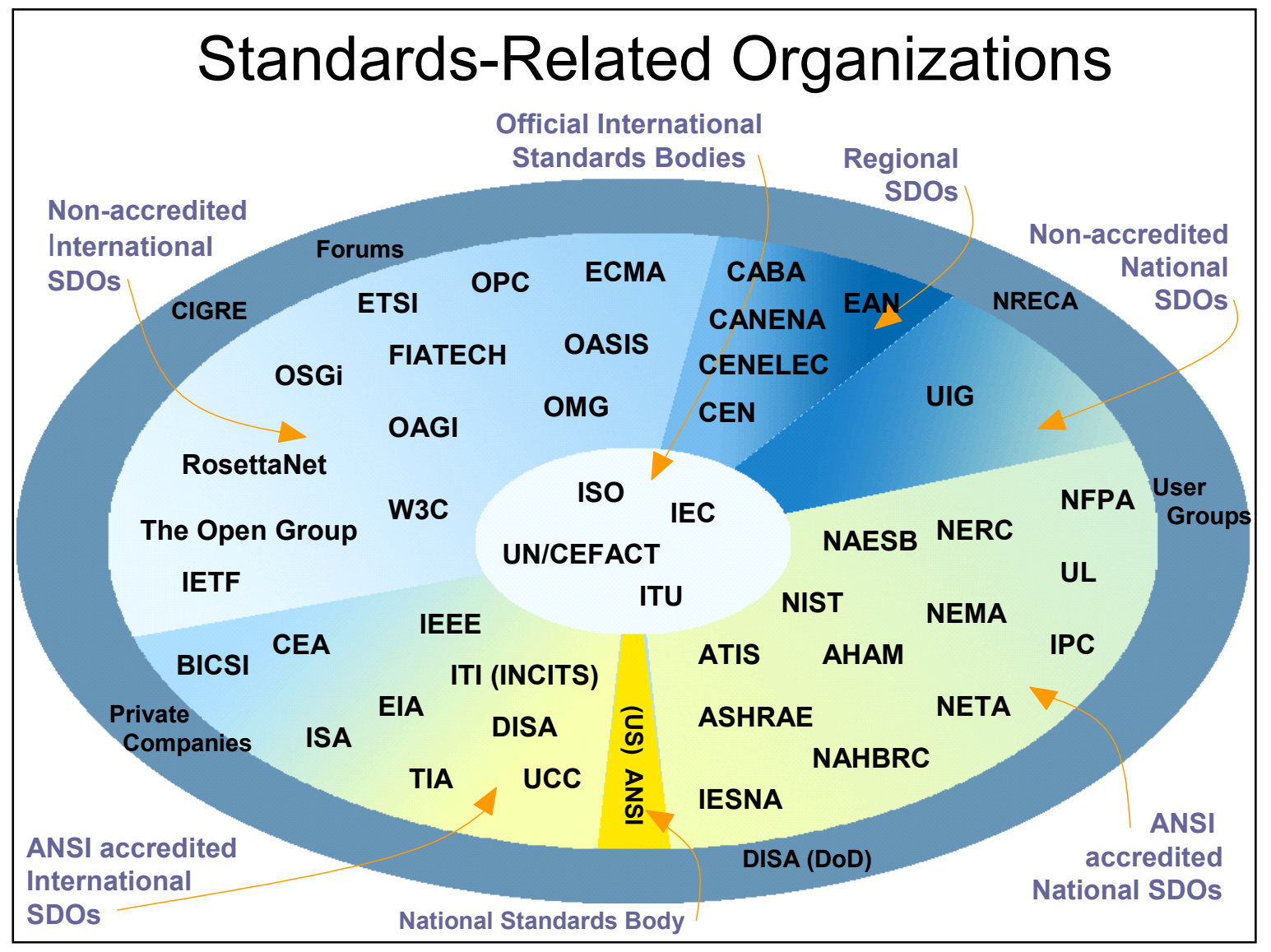

Explanations of acronyms and details on many of these organizations can be found in the alphabetical listing in Appendix A. 